\title{
COMPRESSED ALGEBRAS: ARTIN ALGEBRAS HAVING GIVEN SOCLE DEGREES AND MAXIMAL LENGTH
}

\author{
BY
}

\author{
ANTHONY IARROBINO
}

\begin{abstract}
J. Emsalem and the author showed in [18] that a general polynomial $f$ of degree $j$ in the ring $\mathscr{R}=k\left[y_{1}, \ldots, y_{r}\right]$ has $\left({ }_{r-1}^{j+r-1}\right)$ linearly independent partial derivates of order $i$, for $i=0,1, \ldots, t=[j / 2]$. Here we generalize the proof to show that the various partial derivates of $s$ polynomials of specified degrees are as independent as possible, given the room available.

Using this result, we construct and describe the varieties $G(E)$ and $Z(E)$ parametrizing the graded and nongraded compressed algebra quotients $A=R / I$ of the power series ring $R=k\left[\left[x_{1}, \ldots, x_{r}\right]\right]$, having given socle type $E$. These algebras are Artin algebras having maximal length $\operatorname{dim}_{k} A$ possible, given the embedding degree $r$ and given the socle-type sequence $E=\left(e_{1}, \ldots, e_{s}\right)$, where $e_{i}$ is the number of generators of the dual module $\hat{A}$ of $A$, having degree $i$. The variety $Z(E)$ is locally closed, irreducible, and is a bundle over $G(E)$, fibred by affine spaces $\mathbf{A}^{N}$ whose dimension is known.

We consider the compressed algebras a new class of interesting algebras and a source of examples. Many of them are nonsmoothable-have no deformation to $(k+\cdots+k)$-for dimension reasons. For some choices of the sequence $E, \mathrm{D}$. Buchsbaum, D. Eisenbud and the author have shown that the graded compressed algebras of socle-type $E$ have almost linear minimal resolutions over $R$, with ranks and degrees determined by $E$. Other examples have given type $e=\operatorname{dim}_{k}$ (socle $A$ ) and are defined by an ideal $I$ with certain given numbers of generators in $R=$ $k\left[\left[x_{1}, \ldots, x_{r}\right]\right]$.

An analogous construction of thin algebras $A=R /\left(f_{1}, \ldots, f_{s}\right)$ of minimal length given the initial degrees of $f_{1}, \ldots, f_{s}$ is compared to the compressed algebras. When $r=2$, the thin algebras are characterized and parametrized, but in general when $r>3$, even their length is unknown. Although $k=\mathrm{C}$ through most of the paper, the results extend to characteristic $p$.
\end{abstract}

1. Polynomials having many derivates and compressed algebras. J. Emsalem and the author described in [18] the varieties $G(j)$ (and $Z(j)$ ) parametrizing those graded (or nongraded) Gorenstein Artin algebra quotients $A=R / I$ of the power series ring $R=k[[X]]=k\left[\left[x_{1}, \ldots, x_{r}\right]\right]$ that have the maximum possible length $n=\operatorname{dim}_{k} A$ denoted by $\# A$, given the degree $j$ of the socle of $A$. If $R_{j}$ is the $j$ th graded piece of $R$, it is easy to see that the variety $G(j)$ is naturally an open dense

Received by the editors October 25, 1982 and, in revised form, September 19, 1983.

1980 Mathematics Subject Classification. Primary 13C05; Secondary 14C05, 13E10, 13M10, 13H10, $14 \mathrm{~B} 07$.

Key words and phrases. Artin algebra, Gorenstein algebra, Hilbert function, socle, type, generators, dualizing module, nonsmoothable algebras, minimal resolutions, almost linear resolutions, derivatives of polynomials, linearly independent derivates, maximal rank, zero-dimensional schemes, parametrization, Hilbert scheme, deformation, irreducible, Hankel matrix, cactalecticant, invariants, power sum decomposition, forms, general polynomials, linking, compressed algebra. 
subset of the projective space $\mathbf{P}\left(R_{j}\right)$; then $Z(j)$ is an irreducible variety, a bundle fibred over $G(j)$ by affine spaces $\mathbf{A}^{N(j)}$ of known dimension $N(j)$. The details of this joint result reappear in Theorem I characterizing compressed Gorenstein algebras; the proof here is simpler, and the statement here is slightly stronger than in [18]. These results led to new examples of nonsmoothable Gorenstein algebras-algebras having no deformation to a direct sum of copies of $k$-and thus demonstrated the existence of components of the punctual Hilbert scheme Hilb ${ }^{n^{\prime}} R$ whose general points parametrize nonsmoothable Gorenstein algebras.

Here the results of [18] are extended to Artin algebras $A$ whose type $e(A)$ - the vector space dimension over $k$ of the socle $(0: m)$ of $A$-is greater than one. The nonzero Artin algebra $A$ has socle-type $E=\left(0, e_{1}, \ldots, e_{j}, 0,0, \ldots\right)$ with $e_{j} \geqslant 1$, iff $e_{i}=\#\left(\left((0: m) \cap m^{i}\right) /\left((0: m) \cap m^{i+1}\right)\right)$, where $m$ is the maximal ideal of $A$, and $j$ the smallest integer such that $m^{j+1}=0$. Thus $e_{i}$ is the number of linearly independent initial forms of elements of the socle of $A$ having initial degree $i$, and the type $e(A)=\sum e_{i}$. The Artin algebra $A$ is compressed if it has maximum length among Artin algebras of embedding degree $r$ and of socle type $E(A)$, provided that the socle type $E$ lies among a severely restricted set of sequences called permissible (see Definition 2.2 and the discussion preceding it).

The name compressed algebra $A$ refers to the graph of the Hilbert function sequence $H(A)=\left(h_{0}, h_{1}, \ldots\right)$ where $h_{i}=h_{i}(A)=\# A_{i}$, the length of the $i$ th graded piece of $A$. When $A$ is compressed, the Hilbert function $H(A)$ is maximal: for each $i$, $h_{i}(A) \geqslant h_{i}\left(A^{\prime}\right)$ for $A^{\prime}$ any other Artin algebra of socle type $E(A)$. This maximality of $H(A)$ is shown for graded $A, A^{\prime}$ by Proposition 3.6, which characterizes the Hilbert function of a graded compressed algebra; it is shown for general $A, A^{\prime}$ by $\mathrm{J}$. Elias and the author in [38].

J. Sally has described "stretched Gorenstein algebras" $B$, having in the Artin case minimum length, given the socle degree $j$ and the embedding dimension $r$; their Hilbert function is $H(B)=(1, r, 1, \ldots, 1)$, the minimum possible given $r$ and $j$. In contrast the Hilbert function $H(A)$ of a compressed Gorenstein algebra of socle degree $j$ is symmetric about $j / 2$ and satisfies $h_{i}(A)=\max \left(\# R_{i}, \# R_{j-i}\right)$. For a fixed length $n$, say $n=14$ and $r=3$, a stretched Gorenstein Artin algebra $A$ has Hilbert function $(1,3,1, \ldots, 1)$ most stretched, of maximum possible width $n-r+1=12$; a compressed Gorenstein algebra $A$ has Hilbert function $(1,3,6,3,1)$ most compressed, of minimal width 5 . Thus, the name.

$\$ 2$ gives definitions, examples, and a statement of the main results about families of compressed algebras. $\$ 2 \mathrm{~A}$ presents the well-known duality used in the paper, following J. Emsalem's presentation in [12]. Examples of compressed algebras are listed in $\$ 2 \mathrm{~B}$. The special case, where $A$ is a complete intersection quotient $A=$ $k\left[\left[x_{1}, x_{2}\right]\right] /\left(f_{1}, f_{2}\right)$ of the ring $k\left[\left[x_{1}, x_{2}\right]\right]$ is characterized in $\$ 2 \mathrm{C}$, using power sum decompositions of forms and the Hankel matrix or cactalecticant from classical invariant theory. $\$ 2 \mathrm{D}$ lists the main results of the paper concerning the varieties $G(E)$ and $Z(E)$ parametrizing the compressed, graded and unrestricted (respectively) Artin algebras of socle type $E$. 
The heart of the paper is $\S 3$, giving the proofs, which are elementary and throughout the existence part in $\$ 3 \mathrm{~A}$ are also easily read. The proofs rely on a particularly direct method of constructing the compressed algebras $A$ of socle type $E$, via their dual modules. Their dual modules $V=\operatorname{Hom}(A, k)$ can be naturally identified with those maximal length submodules of the polynomial ring $\mathscr{R}=k[Y]$ $=k\left[y_{1}, \ldots, y_{r}\right]$ that are closed under partial derivation, and which as $R$-modules have $e_{i}$ generators in each degree $i$ : we call these submodules compressed modules (when $E$ is permissible). Here the power series ring $R$ acts as all-order partial differential operators on $\mathscr{R}$.

Definition 1. Action of $R$ on $\mathscr{R}$. If $P \in R=k[[X]]=k \llbracket x_{1}, \ldots, x_{r} \rrbracket$ and $F \in \mathscr{R}=$ $k[Y]=k\left[y_{1}, \ldots, y_{r}\right]$ then $P(F)=P\left(\partial / \partial y_{1}, \ldots, \partial / \partial y_{r}\right) \circ F$. If $S$ is a subset of $\mathscr{R}$, then its annihilator $\operatorname{Ann}(S)$ in $R$ is $\operatorname{Ann}(S)=\{P \in R \mid P(s)=0$ for all $s \in S\}$. If a submodule $S$ of $\mathscr{R}$ is closed under partial differentiation, then $\operatorname{Ann}(S)$ is an ideal of $R$ : we term such submodules $S J$-closed. If $I$ is an ideal of $R$, then its perpendicular set $I^{\perp}$ in $\mathscr{R}$ is $I^{\perp}=\{F \in \mathscr{R} \mid P(F)=0$ for all $P \in I\}$, and it is $J$-closed.

If the algebra $A=R / I$, we identify its dual module $V=\operatorname{Hom}(A, k)$ with $I^{\perp}$ in $\mathscr{R}$. Conversely a $J$-closed vector space $V$ in $\mathscr{R}$ is the dual module to the algebra $A=R / \operatorname{Ann}(V)$. Our insistence here on fixing the dual module $V$ inside $\mathscr{R}$ leads to our parametrization of the compressed algebras $A$ by a variety $Z(E)$.

A key result, determining the length $n(E)$ of the compressed Artin algebras of socle-type $E$, is

Proposition 3.4 (IndePendence OF Higher Derivates). (Assume char $k=0$.) Suppose $F_{1}, \ldots, F_{e}$ are general enough forms of degrees $D=\left(d_{1}, \ldots, d_{e}\right)$ in $\mathscr{R}$. Then for each $s$ with $0 \leqslant s \leqslant \max d_{i}$, the homomorphism $h(s):\left(R_{d_{1}-s}+\cdots+R_{d_{e}-s}\right) \rightarrow \mathscr{R}_{s}$ taking $\left(P_{1}, \ldots, P_{e}\right)$ to $\sum P_{i}\left(F_{i}\right)$ is either injective or surjective.

In other words, the higher partial derivatives of general-enough forms $F_{1}, \ldots, F_{e}$ of fixed degrees $D$ are linearly independent when there is enough room. The forms are general enough when they lie in a certain Zariski-dense subset of the product of projective spaces parametrizing them. The proof of this very natural result uses a consequence of the classical elementary Jordan lemma of invariant theory, that the pure $j$ th powers of linear forms span the vector space $\mathscr{R}_{j}$ of degree-j forms in $\mathscr{R}$. (Note that in Proposition $3.4 R_{-1}=R_{-2}=\cdots=0$.) The proof of Proposition 3.4 can be read in $\S 3 \mathrm{~A}$ independently of the rest of the article. A special case occurs when the degrees are the same $D=(d, \ldots, d)$ : we replace $s$ in Proposition 3.4 by $d-s$ to state it simply.

Proposition 1A (DIFFERENTIAL RELATIONS FOR A SPACE OF DEGREE- $d$ FORMS). If $V$ is a general length l vector space of degree-d forms in $\mathscr{R}_{d}$ (if the point parametrizing $V$ lies in a Zariski-dense subset of the Grassmann variety $\left.\operatorname{Grass}\left(l, \mathscr{R}_{d}\right)\right)$ then the homomorphism $h: R_{s} \otimes V \rightarrow \mathscr{R}_{d-s}$ defined by $h(P \otimes F)=P(F)$, has maximal rank. That is, $h$ is either injective or surjective. 
Proposition 1B (Size of THE QUOTIENT $W: R_{s}$ ). Dually, if the subspace $W \subset R_{d}$ is general enough, then the kernel $\operatorname{Ker} \sigma$ of the homomorphism $\sigma: R_{d-s} \rightarrow$ $\operatorname{Hom}\left(R_{s}, R_{d} / W\right)$ defined by $\sigma(P) \circ(G)=P G \bmod W$, has colength $\operatorname{cod}(\operatorname{Ker} \sigma)=$ $\left(\# R_{d-s}-\# \operatorname{Ker} \sigma\right)$ in $R_{d-s}$ satisfying $\operatorname{cod}(\operatorname{Ker} \sigma)=\max \left(\left(\# R_{s}\right)(\operatorname{cod} W), \# R_{d-s}\right)$. Here Ker $\sigma=R_{-s} W=W: R_{s}=\left\{f \in R_{d-s} \mid R_{s} f \subset W\right\}$, and $\operatorname{cod} W$ is the colength of $W$ in $R_{d}$.

For a proof of Proposition 1A see that of Proposition 3.5 in $\S 3 \mathrm{~B}$. The second part, Proposition 1B, arises from Proposition 1A and the dual viewpoint presented in §2A: the space $W$ is equal to $\operatorname{Ker} \alpha$ where $\alpha$ is the homomorphism $\alpha: R_{d} \rightarrow$ $\operatorname{Hom}(V, k)$ defined by $\alpha(P) \circ F=P(F)$.

EXAMPLE 1.1. Maximal size of $R \circ F$, and compressed Gorenstein algebras. When $r=3$ and $F \in \mathscr{R}$ is a general enough form of degree 4 , then $F ; \partial F / \partial y_{1}, \partial F / \partial y_{2}$, $\partial F / \partial y_{3} ; \partial^{2} F / \partial y_{1}^{2}, \partial^{2} F / \partial y_{1} \partial y_{2}, \ldots, \partial^{2} F / \partial y_{3}^{2}$ are linearly independent; the third partials span $\mathscr{R}_{1}$, and fourth partials span $\mathscr{R}_{0}$. Thus the homomorphism $h$ of Proposition 1A for the space $V=\langle F\rangle$ is injective for $s=0,1,2$ and surjective for $s=2,3,4$. The algebra $A=R /$ Ann $F=R /(P \in R \mid P(F)=0)$ is a compressed Gorenstein algebra of Hilbert function $(1,3,6,3,1)$ (see Figure 1). Example 2.4 in §2B gives a particular form $F$ that is general enough, and specifies the corresponding algebra $A$.

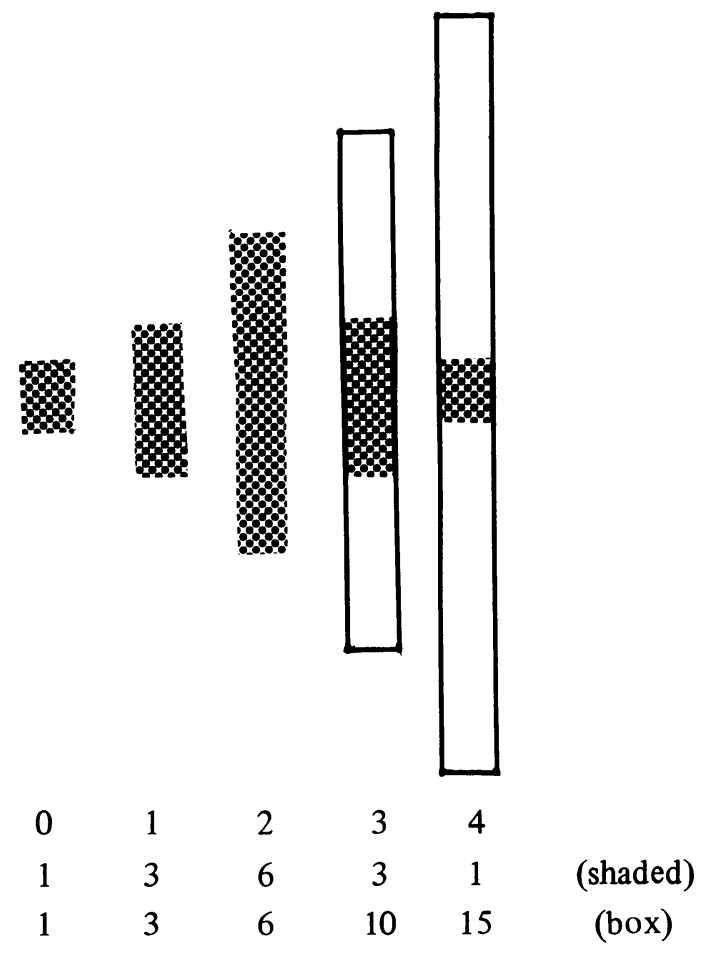

$\begin{array}{ccccccc}\text { Degree } & 0 & 1 & 2 & 3 & 4 & \\ H(A) & 1 & 3 & 6 & 3 & 1 & \text { (shaded) } \\ \# R_{i} & 1 & 3 & 6 & 10 & 15 & \text { (box) }\end{array}$

Figure 1 (EXAmple 1.1). Hilbert function $H(A)$ of a compressed Gorenstein algebra $A$ of socle degree 4. Here $A=R /$ Ann $F$ where $F$ is a general enough form of degree 4 in $\mathscr{R}=k\left[y_{1}, y_{2}, y_{3}\right]$. 


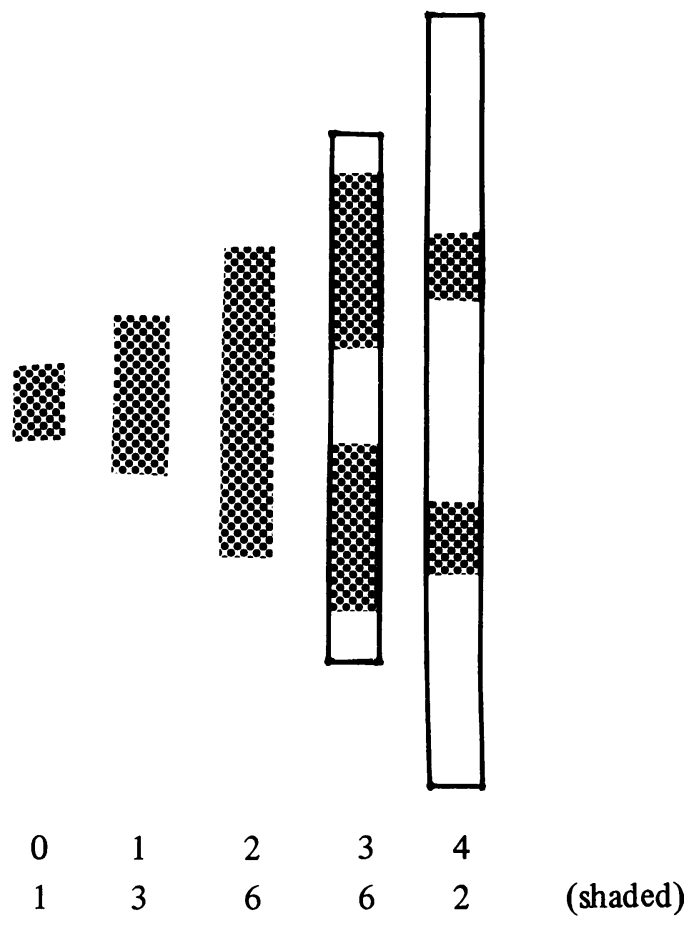

Figure 2 (Example 1.2). Hilbert function $H(A)$ of a compressed type 2 algebra of socle degree 4 . Here $A=$ $R / \operatorname{Ann}(F, G)$ where $F, G$ are general enough forms of degree 4 in $\mathscr{R}=k\left[y_{1}, y_{2}, y_{3}\right]$.

EXAMPLE 1.2. Maximal size of $R \circ\langle F, G\rangle$ and a compressed algebra. When $r=3$ and $F, G$ are general enough forms of degree 4 , then $F, G ; \partial F / \partial y_{1}, \ldots, \partial G / \partial y_{3}$ are linearly independent, while higher partials span $\mathscr{R}_{2}, \mathscr{R}_{1}$, and $\mathscr{R}_{0}$. The homomorphism $h$ for the space $V=\langle F, G\rangle$ is injective for $s=0,1$ and surjective for $s=2,3$, 4. Then $A=R / \operatorname{Ann}(F, G)$ is a compressed Artin algebra of Hilbert function $H(A)=(1,3,6,6,2)$, and socle type $(0,0,0,0,2)$. The dual module $\hat{A}$ equals $J F+J G$, the sum of the dual modules of two usually compressed Gorenstein algebras from Example 1.1. The Hilbert function $H(A)$ is "double" that from Example 1.1. See Figure 2, and also Definition 2.4A in §2D.

A stronger version of Proposition 3.4 is

Proposition 3.5 (INDEPENDENCE OF HIGHER DERIVATES mod A FIXED SPACE). Under the hypotheses of Proposition 3.4, if $V$ is a fixed subspace of $\mathscr{R}_{s}$, the induced homomorphism $\bar{h}(s)=\pi \circ h(s)$ to $R_{s} / V$ is for general $F_{1}, \ldots, F_{e}$ an injection or surjection.

The stronger version permits a step-by-step building of new graded algebras from a given graded algebra $A$ and general enough compressed graded Gorenstein algebras $B_{1}, \ldots, B_{k}$ by adding their dual modules, hence allows us to build graded compressed algebras of arbitrary permissible socle-type $E$ (Proposition 3.6). 
What is the relation between the socle-type of an algebra $A$ and that of $A^{*}$, its associated graded algebra? The algebra $A$ is compressed iff its associated graded algebra $A^{*}$ is compressed; then the socle type $E(A)$ equals $E\left(A^{*}\right)$ (Corollary 3.8 of Proposition 3.7).

Theorems II and III give the dimension and show the irreducibility of varieties $G(E)$ and $Z(E)$ parametrizing graded compressed algebras and general compressed algebras of socle type $E$. They are stated in $\S 2 \mathrm{D}$, and proven in $\S 3 \mathrm{~B}$. The proofs follow directly from the methods of [18] once we know Propositions 3.4-3.7 showing the existence of algebras of socle-type $E$ having the expected maximal length $n(E)$.

$\S 4$ applies these results and gives examples. One example in $\S 4 \mathrm{~A}$ shows that there are generic nonsmoothable Artin algebras of type 2, quotients of $k\left[\left[x_{1}, x_{2}, x_{3}\right]\right]$. In three variables, this is the smallest type possible for nonsmoothable algebras: Kleppe has shown that Gorenstein Artin algebras, quotients of $k\left[\left[x_{1}, x_{2}, x_{3}\right]\right]$, can be smoothed [25].

A second application in $\S 4 \mathrm{~B}$, joint work with D. Buchsbaum and D. Eisenbud, shows that when $E$ satisfies a simple numerical condition, the compressed algebras of socle-type $E$ have almost-linear resolutions (\$4B). This generalizes a special Gorenstein case noted by R. Stanley and also by P. Schenzel [34].

In $\S 4 \mathrm{C}$ we discuss the ideals generated by general enough polynomials of specified orders, and the "thin" algebras they define. This is a different problem, related to that considered hitherto. In the special case $r=2$, we characterize thin algebras, and show for them results analogous to those already shown for compressed algebras (Proposition 4.2). Proposition 4.2 also describes the Hilbert functions possible when $r=2$ for algebras (not necessarily compressed) having socle type $E$. Then what is known about thin algebras when $r \geqslant 3$ is outlined-work of R. Stanley and M. Hochster. Later in $\$ 4 \mathrm{C}$ we answer in the special case $r=2$ variables the following problem of D. Laksov generalizing Proposition 1B: given a general $d$-dimensional vector space $V$ of forms in $R_{j}$, determine the rank of the homomorphism $\theta$ : $R_{s+j-u} \rightarrow \operatorname{Hom}\left(R_{u}, R_{s+j} / R_{s} V\right)$ defined by $\theta(f) \circ g=f g \bmod R_{s} V$. The answer for $r=2$ in Proposition 4.4 is related to the Euclidean algorithm; its proof uses a measure $\tau(V)$ of the complexity of a vector space of forms of the same degree in $R=k\left[x_{1}, x_{2}\right]$.

Which algebras are limits of families of compressed algebras? The first part of $\S 4 \mathrm{D}$ describes when $r=2$ the closures of the families of compressed algebras and of compressed graded algebras of socle type $E$ (Theorem 4.6). These results continue the discussion of Hilbert functions possible for algebras of socle type $E$, begun in Proposition 4.2B of $\S 4 \mathrm{C}$.

A more general class of algebras than "compressed" is the "power sum" algebras, whose dual modules are generated by general sums of a certain number of powers of linear forms. Theorem 4.8 of $\S 4 \mathrm{D}$ extends the existence results of the paper to these algebras.

$\S 4 \mathrm{E}$ extends the results of the paper to the case characteristic $k=p$. In $\S 4 \mathrm{~F}$ some examples illustrate finding compressed algebras having certain specified types $e(A)$ and a specified number of generators $\mu(I)$ for the defining ideal. 
D. Laksov, avoiding duality, has written a concise presentation of Theorem IIB, emphasizing the termwise maximality of the Hilbert function for a compressed algebra; this is the main result of the article in the special case $A$ is graded, and includes the existence of compressed algebras of expected size. His viewpoint is recommended (see $\left.[37]^{1}\right)$. We have retained the dual viewpoint as it forms a natural context for the results even in the special case, and renders more easy their extension to general compressed algebras (Theorem IIC, Proposition 3.7).

A reader wanting a précis of the main results and proofs might follow the introduction by the first part of $\S 2 \mathrm{~A}$, the examples in $\S 2 \mathrm{~B}$, statement of main results in $\S 2 \mathrm{D}$, existence in $\S 3 \mathrm{~A}$, and the key Proposition 3.7 and its Corollary 3.8 of $\S 3 \mathrm{~B}$.

I should like to thank L. Avramov, D. Buchsbaum, D. Eisenbud, D. Berman, J. Weyman, members of the University of Nice Hilbert scheme group, J. Briançon, A. Galligo, M. Granger, J. Spéder, D. Laksov, and members of the Northeastern University Algebra Seminar for their comments. I thank particularly J. Emsalem, with whom I discussed at length the duality used here. I am grateful to D. Buchsbaum and D. Eisenbud for permission to include the joint work of $\S 4 \mathrm{~B}$, and to D. Laksov and R. Stanley for communicating some results in $\S 4 \mathrm{C}$.

\section{What is a compressed algebra?}

2A. Compressed algebras, duality, notation. The duality notation here is adapted from that of $\mathrm{J}$. Emsalem [12]. The ring $R=k[[X]]$ is the power series ring in the variables $X=x_{1}, \ldots, x_{r}$. For simplicity, we assume $k=\mathbf{C}$, the complexes; the case characteristic $k=p$ is discussed in $\S 4 \mathrm{D}$. An Artin ring quotient $A=R / I$ of $R$ has maximal ideal $m=\left(\bar{x}_{1}, \ldots, \bar{x}_{r}\right)$ with $\bar{x}_{i}=x_{i}+I$. The socle $(0: m)$ of $A$ is the subspace $(0: m)=\left\{a \in A \mid x_{i} a=0\right.$ in $A$ for all $\left.i\right\}$. The type $e(A)=\#(0: m)$, the length or vector space dimension over $k$ of the socle.

Definition 2.1. The socle type $E=E(A)=\left(e_{0}, e_{1}, \ldots, 0,0, \ldots\right)$ of the Artin algebra $A=R / I$ is the sequence $E$ of natural numbers where

$$
e_{i}=\#\left(\left((0: m) \cap m^{i}\right) /\left((0: m) \cap m^{i+1}\right)\right) \text {. }
$$

A set $\mathscr{F}=\left\{f_{1}, \ldots, f_{e}\right\}$ of polynomials in $R$ determines a good socle basis $\overline{\mathscr{F}}=$ $\bar{f}_{1}, \ldots, \bar{f}_{e}=f_{1}+I, \ldots, f_{e}+I$ if the vector space span $\langle\bar{F}\rangle=(0: m)$ and if those polynomials of degree $i$ in $\mathscr{F}$ surject to $\left((0: m) \cap m^{i}\right) /\left((0: m) \cap m^{i+1}\right)$.

We now will restrict the sequences $E$ considered for socle types to a small subset termed permissible sequences. Although it would make sense to consider maximal length algebras of any given socle type $E$, their study would require a knowledge of noncompressed Gorenstein algebras-Gorenstein algebras not having maximal length given their socle degree. The compressed algebras-maximal length algebras of permissible socle type $E$-have the property that $I=\bigcap I(i), i=1, \ldots, e(A)$,

\footnotetext{
${ }^{1}$ Note added in proof. R. Fröberg and D. Laksov have extended the notion of compressed to higher dimension algebras $A$ that are graded Cohen-Macaulay; they relate these to the extremal algebras studied by P. Schenzel and J. Sally (see [37]).
} 
where each "factor" $A(i)=R / I(i)$ is a Gorenstein compressed algebra. The decomposition is not unique, but is central to constructing the compressed algebras. The restriction below to permissible sequences $E$ eliminates algebras such as $A=$ $k\left[\left[x_{1}, x_{2}\right]\right] / \operatorname{Ann}\left(y_{1}, y_{2}^{2}\right)=k\left[\left[x_{1}, x_{2}\right]\right] /\left(x_{1} x_{2}, x_{1}^{2}, x_{2}^{3}\right)$ of socle type $E=(0,1,1)$ and Hilbert function $H(A)=(1,2,1)$ : although $A$ has maximal length for its socle type and embedding dimension, it is not compressed (by Definition 2.2(c)). Notice that $A=R / I(1) \cap I(2)$ where $I(1)=\operatorname{Ann}\left(y_{1}\right)=\left(x_{2}, x_{1}^{2}\right)$ defines a compressed Gorenstein algebra, but $I(2)=\operatorname{Ann}\left(y_{2}^{2}\right)=\left(x_{1}, x_{2}^{3}\right)$ defines a noncompressed Gorenstein algebra $A(2)$ of Hilbert function $(1,1,1)$. Since when $r=2$ any Gorenstein compressed algebra $A^{\prime}$ of socle degree 2 has Hilbert function $(1,2,1)$, the same as $H(A)$, such an $A^{\prime}$ cannot be a proper factor of $A$ in the sense above: thus $A$ cannot be decomposed into Gorenstein compressed algebras. However, $A$ is relatively compressed with respect to $A(2)$ - see Definition $2.5 \mathrm{~B}$ in $\S 2 \mathrm{D}$.

Definition 2.2. The sequence $E=\left(0, \ldots, 0, e_{v-1}, e_{v}, \ldots, e_{j}, 0\right)$ is permissible for the socle type of a compressed algebra $A=R / I$ where $I$ has initial degree $v$, and $A$ has top socle degree $j$ (so $I_{j} \neq R_{j}$ and $I_{j+1}=R_{j+1}$ ) if the following conditions are all satisfied:

(a) Each $e_{i} \geqslant 0$, and $e_{j}>0$.

(b) The sum $\sum_{u \geqslant v}\left(\# R_{u-v}\right) e_{u}<\# R_{v}$.

(c) The integer $e_{v-1}=\operatorname{Max}\left(0,\left(\# R_{v-1}-\sum_{u \geqslant v}\left(\# R_{u-(v-1)}\right) e_{u}\right)\right)$.

(b) requires room for generators of $I$ in degree $v$; and (c) assures that there is no room for elements of $I$ in degree $v-1$.

Definition 2.3. Compressed algebra. An Artin algebra $A=R / I$ of permissible socle type $E$ is compressed iff it has maximal length $\# A$ among algebra quotients of $R$ having socle type $E(A)$ and embedding dimension $r$.

For examples, see $\$ 2 \mathrm{~B}$.

Although good socle bases could be used as a unifying concept for the paper, we have chosen to use instead generator sets for the dual module $\operatorname{Hom}(A, k)$. The rest of $\S 2 \mathrm{~A}$ presents this well-known duality (see [25]). A reader trying to get a general idea of the paper may wish to note (d) and (e), then skip to $\S \S 2 \mathrm{~B}$ and $3 \mathrm{~A}$. The duality needed for statements of results is set forth in Definition 1.

The polynomial ring $\mathscr{R}=k[Y]=k\left[y_{1}, \ldots, y_{r}\right]$ acts on $R$ as the algebra of partial differential operators: if $F \in \mathscr{R}, P \in R$, then $F(P)=F\left(\partial / \partial x_{1}, \ldots, \partial / \partial x_{r}\right) \circ P$ is the result. Thus $F=y_{i}^{s}$ acts as $\partial^{s} / \partial x_{i}^{s}$. Similarly, the power series ring $R=k[[X]]$ acts on the polynomial ring $\mathscr{R}$ as partial derivatives, and $P(F)$ denotes the polynomial $P(F)=P\left(\partial / \partial y_{1}, \ldots, \partial / \partial y_{r}\right) \circ F$. To distinguish this latter action of $R$ on $\mathscr{R}$, if $S$ is a subspace of $\mathscr{R}$, we will denote by $J S$ the $R$-submodule of $\mathscr{R}$ generated by $S$. In other words, $J S$ is spanned by the higher partial derivatives of all orders-including 0th order-of elements of $S$. The space $J_{1} S$ is the span of the higher partial derivatives of order at least one of elements of $S$.

The above actions, when composed with evaluation at 0 , make either ring $R$ or $\mathscr{R}$ into a dual of the other. For example, there is an isomorphism $\lambda: R \rightarrow \operatorname{Hom}(\mathscr{R}, k)$ defined by $\lambda(P) \cdot F=P(F)(0)$. Similarly, the ring $\mathscr{R}$ is the ring of continuous linear 
operators on $R$ in the $m$-adic topology. This duality satisfies the following properties (see [25]):

(a) If $M_{P}$ is multiplication by a polynomial $P$ in $R$, the transpose endomorphism of $\mathscr{R}$ is the partial differential operator $P\left(\partial / \partial y_{1}, \ldots, \partial / \partial y_{r}\right)$. Thus, the transpose of $M_{x_{i}}$ is $\partial / \partial y_{i}$. Furthermore, $P(F)(0)=F(P)(0)$.

(b) Passage to the orthogonal $I \rightarrow I^{\perp}=\{P \in \mathscr{R} \mid F(P)(0)=0 \forall F \in I\}$ gives an inclusion-inverting bijection

$$
\begin{gathered}
\tau: \text { ideals } I \text { in } R \Leftrightarrow \text { subspaces } V=I^{\perp} \text { of } \mathscr{R} \text { that are } \\
\text { stable under derivation }(J \text {-closed }) .
\end{gathered}
$$

Furthermore, those ideals of $R$ having finite colength $n$ (with $\#(R / I)=n$ ) are paired by $\tau$ to the length- $n J$-closed subspaces of $\mathscr{R}$. The vector space $V=I^{\perp} \subset \mathscr{R}$ is in a natural way the dual module $V=\operatorname{Hom}(A, k)$ of $A=R / I$. The number of generators of $V$ as an $R$-module, $\#\left(V / J_{1} V\right)=\#\left(V /\left(\left(x_{1}, \ldots, x_{r}\right) \circ V\right)\right)$ is the type $e(A)=\#(0: m)$ of the algebra $A$.

(c) Conversely, given a length- $n J$-closed submodule $V=J S$ of $\mathscr{R}$, generated by $S$, its orthogonal space $I=V^{\perp} \subset R$ is the ideal $I=\{P \mid v(P)(0)=0$ for all $v \in V\}$. By (a) this is just $I=\{P \mid P(v)(0)=0 \forall v \in V\}$ and is easily shown to be $I=\{P \mid P(v)$ $=0 \forall v \in V\}=\{P \mid P(s)=0 \forall s \in S\}$ or in other words $I=\operatorname{Ann}(V)=\operatorname{Ann}(S)$, the annihilator of $V$ or of $S$ in the $J$-action of the power series ring $R$ on the polynomial ring $\mathscr{R}$.

(d) Recall that the Hilbert function $H(A)$ of the Artin algebra $A=R / I$ is the sequence, $H(A)=\left(h_{0}, h_{1}, \ldots, 0,0\right)$ where $h_{i}=\# A_{i}$ and $A_{i}=R_{i} / I_{i}$ is the $i$ th graded piece of $\mathrm{Gr}_{m} A=A^{*}$, the associated graded algebra of $A$. Here

$$
I_{i}=\left(I \cap m^{i}+m^{i+1}\right) / m^{i+1} .
$$

Note that the grading used on $R$ and on $A$ is the initial degree of elements $P \in R$, but that used on $\mathscr{R}=k[Y]$ is the usual degree of elements $v \in \mathscr{R}$. Thus, we define the degree-type or descending Hilbert function $H(V)$ for the finite-length vector subspace $V \subset \mathscr{R}$ to be the sequence $H(V)=\left(h_{0}, h_{1}, \ldots, 0,0, \ldots\right)$ where $h_{i}=\# V_{i}$ and where

$$
V_{i}=\left(V \cap J \mathscr{R}_{i}+J \mathscr{R}_{i-1}\right) /\left(J \mathscr{R}_{i-1}\right) .
$$

Here $V_{i}$ is the $i$ th graded piece of $V$ when the grading is by degree, and $J \mathscr{R}_{i}$ is the space of all polynomials in $R$ having degree at most $i$. When $A=R /$ Ann $V$, then $A_{i}=\operatorname{Hom}\left(V_{i}, k\right)=R_{i} /\left(\right.$ Ann $\left.V_{i} \cap R_{i}\right)$ and the degree-type $H(V)$ satisfies $H(V)=$ $H(R /$ Ann $V)=H(A)$, the Hilbert function of $A$. All our Hilbert function, degreetype, and socle-type sequences end in zeros; the notation $H=\left(h_{0}, \ldots, h_{j}\right)$ stands for $H=\left(h_{0}, \ldots, h_{j}, 0,0, \ldots\right)$.

(e) The generator type $E=E(V)=\left(e_{0}, \ldots, e_{s}\right)$ of a finite $J$-closed subspace $V \subset \mathscr{R}$ is the degree-type $E=H(S)$ of a minimal generating set $S$ for $V$ as a $J$-module. The set $S$ is a minimal generating set if the projection homomorphism $S \rightarrow\left(V / J_{1} V\right)=\left(V /\left(x_{1}, \ldots, x_{r}\right) \circ V\right)$ is a bijection. 
When the Artin algebra $A$ equals $R /$ Ann $V$, the dual generator degrees of $A$ are the sequence $E(V)$, the generator-type of $V$. Each $e_{i}$ is then the number of degree-i dual generators of $A$, or degree- $i$ generators of $V$, or also, as Lemma 2.1 shows, the number of socle elements of $A$ having degree $i$. The number $e_{i}$ depends only on $V$ (or $A$ ) and not on the choice of the generating set $S$ for $V$. The sum $\sum e_{i}$ is the type $e(A)$. Here, if $A=R / I$ is given, the space $V=I^{\perp}$.

Lemma 2.1 (DuAl Generator TYPe EQUALS SOCle TYPe). If $A=R / I$ is an Artin algebra having dual module $V=I^{\perp} \subset \mathscr{R}$ and if $S$ is a minimal generating set for $V$, then the dual-generator degrees of $A$ are $E(V)=H(S)=E(A)$, the socle type of $A$. Moreover, if $N_{i}=\left((0: m) \cap m^{i}\right)$ in $A$, then $\left(N_{i} / N_{i+1}\right)=\hat{S}_{i}=\operatorname{Hom}\left(S_{i}, k\right)$, the dual space to $S_{i}$ in a natural action induced by that of $R$ on $\mathscr{R}$.

Proof. As the socle type of $A$ (Definition 2.1) is the sequence $h_{i}(A)=\#\left(N_{i} / N_{i+1}\right)$, it suffices to prove $\left(N_{i} / N_{i+1}\right)=\hat{S}_{i}$. It is easily seen that $J_{1} V=(0: m)^{\perp}$ and $\left(V \cap J \mathscr{R}_{i-1}\right)=\left(m^{i}\right)^{\perp}$ in the isomorphism $V=\hat{A}=\operatorname{Hom}(A, k)$. Consequently,

$$
N_{i}=\left((0: m) \cap m^{i}\right)=\left(J_{1} V+V \cap\left(J \mathscr{R}_{i-1}\right)\right)^{\perp}=(S(i))^{\wedge},
$$

where

$$
\begin{aligned}
S(i) & =\left(\left(S+J \mathscr{R}_{i-1}\right) /\left(J \mathscr{R}_{i-1}\right)\right)=\left(V+J \mathscr{R}_{i-1}\right) /\left(J_{1} V+J \mathscr{R}_{i-1}\right) \\
& =V /\left(J_{1} V+V \cap\left(J \mathscr{R}_{i-1}\right)\right) .
\end{aligned}
$$

By definition,

$$
S_{i}=\left(\left(S \cap\left(J \mathscr{R}_{i}\right)+J \mathscr{R}_{i-1}\right) / J \mathscr{R}_{i-1}\right)=\operatorname{Ker}(S(i) \rightarrow S(i+1)),
$$

whence $0 \rightarrow S_{i} \rightarrow S(i) \rightarrow S(i+1) \rightarrow 0$ is exact. Dualizing,

$$
0 \rightarrow \operatorname{Hom}(S(i+1), k) \rightarrow \operatorname{Hom}(S(i), k) \rightarrow \operatorname{Hom}\left(S_{i}, k\right) \rightarrow 0
$$

is exact, and $\left(S_{i}\right) \hat{=} \operatorname{cok}\left(N_{i+1} \rightarrow N_{i}\right)=N_{i} / N_{i+1}$, as claimed.

COROLlaRY 2.2A (COMPRESSED ALGEBRAS AND COMPRESSED MODULES). The compressed Artin algebra quotients of $R$ having socle type $E$ correspond one-to-one with the $J$-closed submodules $V \subset \mathscr{R}$ of generator type $E$ and having maximum length.

COROLlARY 2.2B. The length of a compressed algebra of socle type $E$ is the maximal number JS of linearly independent partial derivatives of all orders including 0 of a vector space $S$ of degree-type $E$ in $\mathscr{R}$.

COROLlaRY 2.2C. The family of compressed algebra quotients $A$ of $R$ having socle type $E$ is in one-to-one correspondence with the classes of subspaces $S$ of degree-type $E$ in $\mathscr{R}$ such that $J S$ has maximal length, $\bmod$ the equivalence $S \equiv S^{\prime}$ if $J S=J S^{\prime}$.

2B. Examples of compressed algebras. The following examples are intended to give an intuitive idea of compressed algebras. The notation is that of Definition 1 in $\S 1$, further explained in $\$ 2 \mathrm{~A}$. 
EXAMPLE 2.1. Compressed complete intersection. In two variables, the $J$-submodule of $\mathscr{R}=k\left[y_{1}, y_{2}\right]$ generated by the form $F=\left(y_{1}^{3}+y_{2}^{3}\right)$ is $V=J F=$ $\left(F, y_{2}^{2}, y_{3}^{2}, y_{2}, y_{1}, 1\right)$ of length 6 with $H(V)=(1,2,2,1)$. The module $V$ is dual to the complete intersection (c.i.) algebra $A=R / I$ with $I=\operatorname{Ann} V=\left(x_{1} x_{2}, x_{1}^{3}-x_{2}^{3}\right)$. Since a form $F$ of degree 3 in $R$ can have at most 2 first partials, 2 second partials (of degree 1 ), and 1 third partial of degree 0 , the $J$-module $V$ has maximum possible length six, given its generator type $E(V)=(0,0,0,1)$ and embedding dimension two. Thus the algebra $A=k\left[\left[x_{1}, x_{2}\right]\right] /\left(x_{1} x_{2}, x_{1}^{3}-x_{2}^{3}\right)$ is a compressed algebra of socle type $E(A)=(0,0,0,1)$.

EXAMPLE 2.2. Complete intersection, not compressed. If we choose instead $f=\left(y_{1}^{2}\right.$ $\left.+y_{2}^{3}\right)$ then $V=J f=\left(f, y_{2}^{2}, y_{2}, y_{1}, 1\right)$ of length 5 (nonmaximal) and degree-type $H(V)=(1,2,1,1)$, corresponding to the c.i. $A=R / I$ with $I=\left(3 x_{1}^{2}-x_{2}^{3}, x_{1} x_{2}\right)$, which is not compressed. It is easy to see that when $r=2$, the degree 3 polynomial $f$ generates a compressed c.i. iff the top degree form $F$ of $f$ is not a perfect cube $F=\left(a y_{1}+b y_{2}\right)^{3}$.

EXAMPLE 2.3. Complete intersection, compressed. If $r=2$, and $F=y_{1}^{4}+y_{2}^{4}+$ $\left(y_{1}+y_{2}\right)^{4}$ then

$$
V=J F=\left(F, y_{1}^{3}+\left(y_{1}+y_{2}\right)^{3}, y_{2}^{3}+\left(y_{1}+y_{2}\right)^{3}, y_{1}^{2}, y_{1} y_{2}, y_{2}^{2}, y_{1}, y_{2}, 1\right) \text {. }
$$

The ideal $I=$ Ann $V=\left(\left(x_{1}^{2} x_{2}-x_{1} x_{2}^{2}\right),\left(x_{1}^{3}+x_{2}^{3}-3 x_{1}^{2} x_{2}\right)\right)$, defines a graded complete intersection algebra $A=R / I$ of Hilbert function $H=(1,2,3,2,1)$. It has maximum length 9 , given the socle degree 4 and embedding dimension $r=2$, so is compressed. Beginning anew with $f=F+h$, where $h$ is a polynomial of degree 3 , will lead to a compressed complete intersection algebra $A^{\prime}=R / \operatorname{Ann}(f)$, in general not graded, whose associated graded algebra is $A$. This example illustrates the fact that an Artin algebra is compressed iff its associated graded algebra is compressed (Corollary 3.8).

There is a complete description of compressed complete intersection algebras when $r=2$ in Lemma 2.3 of $\$ 2 \mathrm{C}$. The simplest case occurs when the form $F$ determining $V$ is the sum of $t+1$ perfect $j$ th powers of linear forms, $F=\sum_{i=1}^{t+1} a_{i} L_{i}^{j}$ with $0 \neq a_{i} \in k$, where $j=2 t$ or $2 t+1$. Then $A=R /$ Ann $F$ is compressed. In the example above, $j=4$, and $F$ is a sum of 3 perfect 4 th powers of linear forms.

In more than two variables, the only compressed complete intersection algebras are those with socle degree $j=1$, or certain algebras where $H(A)=(1,3,3,1)$ when $r=3$. The non-c.i. compressed Gorenstein algebras have defining ideals $I=$ Ann $f$ with many generators. The number of generators is exactly $\left(\# R_{t+1}-\# R_{t-1}\right)$ when $j$ is even and $t=j / 2$, and is at least $\left(\# R_{t+1}-\# R_{t}\right)$ when $j$ is odd and $t=(j-1) / 2$. See also Theorem I and Example 4.7 in $\S 4 \mathrm{~B}$.

EXAMPLE 2.4. Compressed Gorenstein algebra. When $r=3$ the $J$-module $V=J F$ generated by $F=y_{1}^{4}+y_{2}^{4}+y_{3}^{4}+\left(y_{1}+y_{2}\right)^{4}+\left(y_{1}+y_{3}\right)^{4}+\left(y_{2}+y_{3}\right)^{4}$ has degree-type $H(V)=(1,3,6,3,1)$ of length 14: its annihilator is an ideal $I$ with 7 generators of degree 3 , namely $I=\left(f_{1}, \sigma f_{1}, \sigma^{2} f_{1}, f_{2}, \sigma f_{2}, \sigma^{2} f_{2}, f_{3}\right)$ where $f_{1}=x_{1}^{2} x_{2}$ $-x_{1} x_{2}^{2}, f_{2}=2 x_{1}^{2} x_{2}-2 x_{1}^{2} x_{3}+x_{3}^{3}-x_{2}^{3}, f_{3}=\left(1+\sigma+\sigma^{2}\right) \cdot\left(2 x_{1}^{3}-5 x_{1}^{2} x_{2}\right)$ and $\sigma$ denotes the cyclic permutation $\left(x_{1}, x_{2}, x_{3}\right)$. Since $e(A)=1$ and $F$ is a homogeneous 
form, the algebra $A=R / I$ is Gorenstein and graded; since a degree-4 form $F$ can have at most 3 first, 6 second, and 3 third partials, the module $J F$ has the maximum possible length, and $A$ is compressed. Beginning anew with $f=F+h$ (with $h$ of degree 3 ) leads to other compressed but usually nongraded Gorenstein algebras $A^{\prime}$ having the algebra $A$ as associated graded algebra.

EXAMPLE 2.5. Compressed algebra of type $e(A)=2$. In 3-variables, the $J$-module $V=J S$ where $S=\left(F, y_{1}^{3}\right)$ and $F$ is from Example 2.4, satisfies $H(V)=(1,3,6,4,1)$ and is the dual module $V=\hat{A}$ to a compressed algebra $A=R / I$ of type 2 defined by an ideal $I=\operatorname{Ann}(S)$ having 6 generators, all of degree 3, namely $I=$ $\left(f_{1}, \sigma f_{1}, \sigma^{2} f_{1}, f_{2}, \sigma f_{2}+\sigma^{2} f_{2}, f_{3}-2 \sigma f_{2}\right)$ in the notation of Example 2.4. Here the socle type $E(A)=(0,0,0,1,1)$.

Note. For a complete description of compressed algebras of embedding dimension $r=2$ and type $e(A) \geqslant 2$ see Proposition 4.2B in $\S 4 \mathrm{C}$. The Gorenstein (c.i.) case $e(A)=1$ and $r=2$ follows now.

2C. Compressed complete intersection algebras when $r=2$ : power sum decompositions and the cactalecticant. Lemma 2.3 generalizes Example 2.3. It characterizes compressed complete intersection algebras when $r=2$, in terms of the decomposition $F=\sum B_{i} L_{i}^{j+1-s_{i}}$ of the top degree form $F$ of a generator $f$ for the dual module of $A$ into sums of powers of linear forms. In the notation of Example 2.3, Lemma 2.3 would imply that the form $F^{\prime}=y_{1}^{4}+y_{2}^{3}\left(y_{1}+y_{2}\right)$ also determines a graded compressed complete intersection algebra having Hilbert function $H=(1,2,3,2,1)$, since $F^{\prime}$ is a generalized sum of 3 fourth powers.

Below, the form $F$ of degree $j$ in $k\left[y_{1}, y_{2}\right]$ is a generalized sum of $s$ jth powers of linear forms iff $F=\sum B_{i} L_{i}^{j+1-s_{i}}$ where each $B_{i}$ is a (coefficient) form of degree $s_{i}-1$, each $L_{i}=\left(a_{i} y_{i}+a_{i}^{\prime} y_{2}\right)$ is a linear form, $s=\sum s_{i}$ and no $L_{i}$ depends on $L_{i}$, for $i^{\prime} \neq i$. In the case $A$ is graded ( $f=F$ ) Lemma 2.3 is classical, due to the theory of apolar forms $[16, \S 189]$ and to Macaulay's result that a Gorenstein algebra $A=R / I=k\left[\left[x_{1}, x_{2}\right]\right] / I$ is a complete intersection [26] (see also [24]). Below we denote by $H_{0}$ the sequence $H_{0, t}=(1,2, \ldots, t, t+1, t, \ldots, 1)$ of length $(t+1)^{2}$ when $j$ is even, or the sequence $H_{0, t}^{\prime}=(1,2, \ldots, t+1, t+1, \ldots, 1)$ of length $(t+1)(t+2)$ when $j$ is odd.

Lemma 2.3 ((F. S. Macaulay et Al.) Compressed complete intersection ALGEBRAS WHEN $r=2$ : SUMS OF POWERS AND APOLAR FORMS). When $r=2$, and $f$ is a polynomial in $R$ of degree $j=2 t$ (or $j=2 t+1$, respectively) then TFAE:

(A) The complete intersection algebra $A=R / \operatorname{Ann}(f)$ is compressed.

(B) The Hilbert function $H(A)$ equals the sequence $H_{0}$.

(C) The top degree form $F$ of $f$ can be written as a generalized sum of $(t+1)$ jth powers of linear forms with each coefficient $B_{i} \neq 0$. Equivalently, $F$ is not a generalized sum of only $t$ th powers.

Proof of (A) $\Leftrightarrow$ (B). It is not hard to show that $\#\left((J f)_{i}\right) \leqslant \min \left(\# R_{i}\right.$, \#R $\left.R_{j-i}\right)$. When $f=F$ is graded the inequality is obvious; the nongraded case follows from Macaulay's result that Gorenstein implies c.i. when $r=2$ and from Macaulay's restriction on the sequences possible for Hilbert functions of c.i. quotients of $R$. 
These results imply $H(R / \operatorname{Ann} f)=\left(1,2, \ldots, d, h_{d}, \ldots, h_{j}=1,0\right)$ with $h_{i}-h_{i-1}=0$ or 1 for each $i$ (see [27]; in [21; 23, Appendix Theorem A1, or 8] these results are generalized). It follows that $h_{i} \leqslant \min \left(\# R_{i}, \# R_{j-i}\right)$. Thus, $\# A=\sum \#\left((J f)_{i}\right) \leqslant$ $\sum \min \left(\# R_{i}, \# R_{j-i}\right)=(t+1)^{2}$ or $(t+1)(t+2)$ respectively (when $j$ is even or odd), with equality iff $H(A)=H_{0}$ is the sequence specified in Lemma 2.3(B). The equivalence $(\mathrm{A}) \Leftrightarrow(\mathrm{B})$ then follows from the existence-shown below-of graded algebras $A=R /$ Ann $F$ having the Hilbert function $H(A)=H_{0}$.

To show (B) $\Leftrightarrow(C)$ (and thus to complete the proof of (A) $\Leftrightarrow(B)$ ) we need a well-known result from the classical theory of apolar forms.

Claim. Apolar forms of $F$. The form $F \in \mathscr{R}$ is a generalized sum $F=\sum B_{i} L_{i}^{j+1-s_{i}}$ of $s j$ th powers where $s \leqslant j+1, L_{i}=\left(a_{i} y_{1}+a_{i}^{\prime} y_{2}\right)$, and $L_{i} \neq L_{i^{\prime}}$ when $i \neq i^{\prime}$, if and only if the graded ideal $I^{*}=$ Ann $F \subset R$ contains the form $P=\Pi\left(\bar{L}_{i}\right)^{s_{i}}$, where $\bar{L}_{i}=\left(a_{i}^{\prime} x_{1}-a_{i} x_{2}\right)$. Here $s=\sum s_{i}$.

Proof OF CLAIM. The form $\bar{L}_{i}^{s_{i}}$ acting on $\mathscr{R}$ vanishes on $\mathscr{R}_{s_{i}-1} L_{i}^{u}$ for any integer $u$; hence the subspace $W=\oplus R_{s_{i}-1} L_{i}^{j+1-s_{i}}$ of $\mathscr{R}_{j}$ satisfies $W \subset \operatorname{Ker} P$. To prove the claim we need to show $W=(\operatorname{Ker} P) \cap \mathscr{R}_{j}$. The functional $\bar{L}_{i}$ maps $\mathscr{R}_{u}$ onto $\mathscr{R}_{u-1}$ (consider the functional $x_{1}$, acting as $\partial / \partial y_{1}$ on $\mathscr{R}_{u}$ ); thus $P$ maps $\mathscr{R}_{j}$ onto $\mathscr{R}_{j-s}$. It follows that $\#\left((\operatorname{Ker} P) \cap \mathscr{R}_{j}\right)=\left(\# R_{j}-\# R_{j-s}\right)=s$, which is $\# W$ by Lemma 3.1. The equality of dimensions shows that the sum $W=\oplus$ is direct and that $W=$ $(\operatorname{Ker} P) \cap \mathscr{R}_{j}$, as claimed.

Proof OF (B) $\Leftrightarrow(C)$. It is easy to show that for any form $F \in \mathscr{R}_{j}$, the c.i. ideal Ann $F=\left(G_{1}, G_{2}\right)$ in $R$ has generator degrees $\left(v_{1}, v_{2}\right)$ satisfying $v_{1} \leqslant v_{2}$ and $v_{1}+v_{2}$ $=j+2$. Then the Hilbert function

$$
H(R / \text { Ann } F)=\left(1,2, \ldots, h_{v_{1}-1}=v_{1} ; v_{1}, \ldots ; h_{v_{2}}=v_{1}-1 ; v_{1}-2, \ldots, 1,0\right) .
$$

Thus, the condition (C) is satisfied iff the initial degree of $I^{*}=$ Ann $F$ is $v_{1}=t+1$, or equivalently if $H(R / \operatorname{Ann} F)=H_{0}$, the Hilbert function in Lemma 2.3(B). This fact shows (B) $\Leftrightarrow(\mathrm{C})$ in the graded case $f=F$. It also shows the existence of graded algebras of Hilbert function $H_{0}$ (choose $F$ the sum of $t+1 j$ th powers), so completes the proof of $(\mathrm{A}) \Leftrightarrow(\mathrm{B})$. Corollary 3.8 implies that in general $A=R / \operatorname{Ann} f$ is compressed iff the associated graded algebra $A^{*}$ is compressed; then $A^{*}=R / \operatorname{Ann} F$ where $F$ is the top degree form of $f$. This equivalence and (A) $\Leftrightarrow$ (B) complete the proof of $(B) \Leftrightarrow(C)$ and of the lemma.

Note. The cactalecticant is the determinant $D(F)$ that vanishes when the space $J^{t} F$ has less than maximal rank $\# R_{t}$, when the degree $j$ of the form $F$ is even [16, §187]. Thus, when $j=2 t, J F$ is the dual module to a compressed Gorenstein algebra iff $D(F)$ is nonzero. When $r=2$, this occurs when $F$ is not a generalized sum of $t j$ th powers of linear forms: then the c.i. ideal $I=$ Ann $F$ has two generators, both of degree $t+1$. The case $r=2$ of the cactalecticant has been much studied under the aliases Hankel, or persymmetric determinant.

Theorem I (Gorenstein compressed algebras) of this paper is Theorem 3.31 in [18]. The proof of the even $j=2 t$ case in [18] uses induction on $r$ to show that $F$ can be chosen so that the $r$-variable cactalecticant $D(F)$ is nonzero. The proof of Theorem I 
here also uses the cactalecticant: the determinant of the matrix $M$ in the proof of Proposition 3.4 is $D(F)$ for a particularly well chosen form $F$. In both proofs the nonvanishing of a cactalecticant is central. However, the power-sum decomposition interpretation of the cactalecticant in Lemma 2.3 is valid only when $r=2$.

2D. Results for compressed algebras. This section states the results concerning compressed Artin algebras; these results are restated and are proven in $\S 3$, using the language of dual modules. A reader wanting to get a quick idea of main results and method may wish to skim this section and proceed to the heart of the paper, §3A. The first main result is the following theorem about compressed Gorenstein algebras $(e(A)=1)$ shown jointly with J. Emsalem [18]. Recall that the variety $G(j)$ (or $Z(j)$, respectively) parametrizes graded (or all, respectively) Gorenstein algebras $A$ of maximal length $n(j)$-defined below-having socle-degree $j$. These are algebras of socle-type $E(A)=(0,0, \ldots, 0,1)$ with 1 in degree $j$ only. Recall that the family of length- $n$ algebra quotients of $R$ has a natural scheme structure $\operatorname{Hilb}^{n} R$; thus $Z(j)$ defines a locally closed subset of $\operatorname{Hilb}^{n} R$ and receives the reduced subscheme structure. The scheme structures on $Z(E), G(E), Z(A ; E), G(A ; E)$ that we discuss later in the section, are similarly defined.

TheOREM I (COMPRESSED GORENSTEIN ALGEBRAS). The variety $G(j)$ is an open dense subset of the projective space $\mathbf{P}\left(R_{j}\right)$; the variety $Z(j)$ is a bundle fibred over $G(j)$ by an affine space $\mathbf{A}^{N(j)}$. When $j=2 t+1$ is odd, then the Hilbert function $H(j)$ of these compressed algebras is

$$
H(j)=\left(\# R_{0}, \# R_{1}, \ldots, \# R_{t}, \# R_{t}, \ldots, \# R_{1}, 1\right)
$$

of length $n(j)=\sum h_{i}=2\left(\begin{array}{c}r+t \\ t\end{array}\right)$. Then $\operatorname{dim} G(j)=\# R_{j}-1$, and

$$
\operatorname{dim} Z(j)=\sum_{t+1}^{j}\left(\# R_{i}-h_{i}\right)=\sum_{t+1}^{j}\left(\# R_{i}-\# R_{j-i}\right)=\left(\begin{array}{c}
r+j \\
j
\end{array}\right)-2\left(\begin{array}{c}
r+t \\
t
\end{array}\right) .
$$

When $j=2 t$ is even, then the Hilbert function is $H(j)=$ $\left(\# R_{0}, \ldots, \# R_{s}, \# R_{s-1}, \ldots, \# R_{1}, 1\right)$ of length $n(j)=2\left(\begin{array}{c}r+t \\ t\end{array}\right)-\left(\begin{array}{c}r+t-1 \\ t\end{array}\right)$; and the dimension

$$
\begin{aligned}
\operatorname{dim} Z(j) & =\sum_{t+1}^{j}\left(\# R_{i}-h_{i}\right)=\sum\left(\# R_{i}-\# R_{j-i}\right) \\
& =\left(\left(\begin{array}{c}
r+j \\
j
\end{array}\right)-2\left(\begin{array}{c}
r+t \\
t
\end{array}\right)+\left(\begin{array}{c}
r+t-1 \\
t
\end{array}\right)\right) .
\end{aligned}
$$

Note. Since Proposition 3.7 shows that a compressed algebra has compressed associated graded algebra, Theorem $I$ is more complete here than in [18]; the statement above corrects a typographic error of [18] in $\operatorname{dim} Z(j)$ when $j$ is odd. The existence of compressed Gorenstein algebras of length $n(j)$ and socle degree $j$ is also independently shown by E. L. Green in [15] by a method similar in style to ours in [18]. Also, P. Schenzel studied these "extremal Gorenstein algebras" when the socle 
degree $j$ is even; he noted that work of combinatorialists R. McMullen, R. Stanley, and of M. Hochster leads to explicit examples [34, Beispiele 4.7]. The proof of the existence of Gorenstein compressed algebras here in Proposition 3.3, although not constructive, is different and simpler than the earlier methods. Once one knows that there is a compressed algebra $A$ of specified socle-type $E$ and having the expected maximal length $n(E)$ (here $n(j)$ ), then the dimension and structure of $Z(E)$ and $G(E)$ (here $Z(j)$ and $G(j)$ ) follow from our method in $\S 3$ of [18]. We need now a notation for the Hilbert function $H(E)$ of a compressed algebra of socle type $E$.

Definition 2.4A. The sum Hilbert function $H^{\prime \prime}=H+H^{\prime}$ is the sequence $H^{\prime \prime}=$ $\left(h_{0}^{\prime \prime}, h_{1}^{\prime \prime}, \ldots\right)$ where $h_{i}^{\prime \prime}=\min \left(\# R_{i}, h_{i}+h_{i}^{\prime}\right)$.

Definition 2.4B. If $E$ is a permissible socle type sequence, (Definition 2.2) then the Hilbert function sequence $H(E)$ is $\sum e_{u} H(u)$, the sum in the above sense of Hilbert functions $H(u)$ of compressed Gorenstein algebras (Theorem I). Thus $H(E)=\left(h_{0}, h_{1}, \ldots\right)$ with $h_{i}=\sum_{u \geqslant i} e_{u}\left(\# R_{u-i}\right)$ if $i \geqslant v$, and $h_{i}=\# R_{i}$ if $i<v$. Let $n(E)=\sum h_{i}$.

Theorem IIA(Proposition 3.4) COMPRESSED ALGEBRAS AND COMPRESSED MODULES). If $E$ is a permissible sequence, (Definition 2.2) then the sequence $H(E)$ is the Hilbert function of each compressed algebra having socle-type $E$. Such algebras $A$ have length $n(E)$ and have the form $A=R / \operatorname{Ann}(V)$ where $V=J S$, with $S$ a general enough submodule of $\mathscr{R}=k[Y]$ having degree-type $E$. The "compressed" module $V$ is uniquely determined by $A$; conversely, if the subspace $S \subset \mathscr{R}$ has degree type $E$, and if $V=J S$ satisfies $\# J S=n(E)$, then $A=R / A n n(V)$ is a compressed algebra of socle-type $E$.

Theorem IIB ((Dual version of Proposition 3.6) Family of GRaded COMPRESSED ALGEBRAS OF SOCLE TYPE $E$ ). The graded compressed algebras of socle type $E$ correspond one-to-one with graded submodules $V=J S \subset \mathscr{R}$, where $S$ is a graded module of degree-type $E$. When $S$ is graded, let $S_{>i}$ denote $S_{i+1}+S_{i+2}+\cdots$, let $E_{>i}=\left(0, \ldots, 0, e_{i+1}, e_{i+2}, \ldots\right)$, and denote by $G(E)$ the variety parametrizing graded compressed algebras of degree type $E$. There is a surjective morphism from $G\left(E_{\geqslant i}\right)=$ $G\left(E_{>(i-1)}\right)$ to $G\left(E_{>i}\right)$, having a fibre $F_{p}$ over the point $p$ parametrizing an algebra $A=R /\left(\right.$ Ann $\left.J S_{>i}\right)$. The fibre $F_{p} \subset \operatorname{Grass}\left(e_{i}, \mathscr{R}_{i} /\left(J S_{>i} \cap \mathscr{R}_{i}\right)\right)$ is the open dense subset of the Grassmannian, parametrizing those $e_{i}$-dimensional subspaces $W_{i}$ of $R_{i} /\left(J S_{>i} \cap \mathscr{R}_{i}\right)$ such that the module $\left(J W_{i}+J S_{>i}\right)$ has maximal length. This shows $G(E)=G\left(E_{\geqslant 0}\right)$ is irreducible; in fact, $G(E)$ is isomorphic locally to opens in an affine space $\mathbf{A}^{g(E)}$ of dimension

$$
g(E)=\operatorname{dim} G(E)=\sum\left(e_{u}\right)\left(\# R_{u}-h_{u}\right) .
$$

Theorem IIC ((Propositions 3.9, 3.11) FAMILY of COMPRESSED ALGEBraS AS BUNDLE). The variety $Z(E)$ parametrizing compressed algebras of socle type $E$ is a bundle fibred over $G(E)$. The fibre is an affine space $\mathbf{A}^{N(E)}$ of dimension

$$
N(E)=\operatorname{dim} \text { fibre }=\sum_{i} \sum_{u>i}\left(e_{u}\right)\left(\# R_{i}-h_{i}\right) .
$$

Thus $z(E)=\operatorname{dim} Z(E)=\sum_{i} \sum_{u \geqslant i}\left(e_{u}\right)\left(\# R_{i}-h_{i}\right)$. 
Note. The sums $g(E)$ and $z(E)$ count the distinct ways of choosing parameters for either graded or general subspaces $S$ of degree type $E$ modulus the equivalence $S \equiv S^{\prime}$ if $J S=J S^{\prime}$. For proofs see Corollary 2.2C, Propositions 3.3, 3.4, 3.6, 3.9 and 3.11, and the remark following Proposition 3.11.

The last result concerns algebras $A_{S}$ constructed from a given Artin algebra $A=R / I$ by adding to its dual module $V$, the dual modules $V^{\prime}=J S$ of compressed algebras $A^{\prime}=R / I^{\prime}$ of given socle type $E$. Here $S$ is a subspace of $R$ having degree-type $E$, and $A_{S}=R / I \cap I^{\prime}$.

DEFINITION 2.5A. The sequence $E$ is permissible for the degree-type of the space $S$ in $A_{S}$ if $E$ is permissible (Definition 2.3), and if for all $i, e_{i} \neq 0$ implies $h_{i}(A)+$ $h_{i}(E) \leqslant \# R_{i}$. The Hilbert function $H(A ; E)=H(A)+H(E)$ (see Definition 2.4), and $n(A ; E)=\sum h_{i}(A ; E)$, the length of $H(A ; E)$.

Definition 2.5B. An algebra $A_{S}$ is compressed relative to the Artin algebra $A$ and the sequence $E$ if $E$ is permissible as above, if $S$ has degree type $E$, if $A_{S}$ has length $\# A_{S}=n(A ; E)$, and if $A_{S}$ has dual module $\hat{A}=V^{\prime \prime}=V+V^{\prime}$ as above, where $V=I$ and $V^{\prime}=J S$. Then $A=R / \operatorname{Ann}\left(V^{\prime \prime}\right)$, and $H\left(A_{S}\right)=H(A ; E)$. Equivalently, $A_{S}$ is relatively compressed iff $A_{S}=R / I \cap I^{\prime}$ with $A^{\prime}=R / I^{\prime}$ of socle type $E$, and $\# A_{S}=n(A ; E)$.

TheOREM III (FAMILy of RELATIVELy COMPRESSED ALGEBRAS). Suppose $A$ is $a$ specified Artin algebra, and $E$ is permissible for $S$ as above. Then:

(A) For a Zariski-open family of the subspaces $S$ of $R$ having degree-type $E$, the algebra $A_{S}$ has length $n(A ; E)$, so is compressed relative to $A$.

(B) The variety $G(A ; E)$ parametrizing distinct algebras $A_{S}$, compressed relative to $A$, such that $S$ is graded of degree-type $E$, is an irreducible variety, and is locally an affine space $\mathbf{A}^{g(A ; E)}$, of dimension $g(A ; E)=\sum\left(e_{u}\right)\left(\# R_{u}-h_{u}(A ; E)\right)$.

(C) The variety $Z(A, E)$ parametrizing distinct algebras $A_{S}$ that are compressed relative to $A$, is a bundle fibred over $G(A ; E)$ by an affine space, and the dimension $z(A ; E)=\operatorname{dim} Z(A ; E)=\sum_{i} \Sigma_{u \geqslant i}\left(e_{u}\right)\left(\# R_{i}-h_{i}(A ; E)\right)$.

Note A. The projection $\pi: Z(A ; E) \rightarrow G(A ; E)$ is induced by the map $S \rightarrow S^{*}$ taking $S$ to its associated graded module $S^{*}=\oplus S_{i}$ in $R$ (see $\S 2 \mathrm{~A}(\mathrm{~d})$ ). When $A$ is not graded $G(A ; E)$ can parametrize nongraded algebras.

Note B. The variety $G(E)$ or $Z(E)$ is not usually closed in $\operatorname{Hilb}^{n(E)} R$ or in $\operatorname{Grass}\left(n(E), J \mathscr{R}_{j}\right)$ (see §4D for a discussion).

\section{Linear independence of higher derivates of polynomials.}

3A. Existence of forms with many derivates. This section, the heart of the paper, contains the proofs of the results announced in $\S 1$, concerning the existence of sets $S$ of forms in $\mathscr{R}=k[Y]=k\left[y_{1}, \ldots, y_{r}\right]$ having specified degree-type $H(S)=E$, and such that the vector space $V=J S$ of partial derivatives of all orders of the forms in $S$ satisfies $\# V=n(E)$, the expected maximum size (see Definition 2.4). These results show the existence part of Theorems I-III in $\$ 2$ concerning a compressed algebra $A=R / \operatorname{Ann}(S)$ of socle type $E$, for which $V$ is the dual module.

Recall that if $V$ is a subspace of $\mathscr{R}$, then $V_{i}$ is the $i$ th graded piece of $V$, in the (descending) grading by degree (see (1) in $\$ 2 \mathrm{~A}(\mathrm{~d})$ ); and the degree-type $H(V)$ is the 
sequence $H(V)=\left(h_{0}, h_{1}, \ldots\right)$ where $h_{i}=\operatorname{dim}_{k} V_{i}$. For example, if $f=y_{1}^{3}+y_{2}^{2}$ then the space $V=J f$ is $V=\left\langle f, y_{1}^{2}, y_{1}, y_{2}, 1\right\rangle$; the graded pieces of $V$ are $V_{3}=\left\langle y_{1}^{3}\right\rangle$, $V_{2}=\left\langle y_{1}^{2}\right\rangle, V_{1}=\left\langle y_{1}, y_{2}\right\rangle, V_{0}=\langle 1\rangle$; the associated graded module to $V$ is $V^{*}=$ $\oplus V_{i}$. For simplicity, we assume char $k=0$.

The starting point is a classical Jordan's lemma of invariant theory in two variables. Lemma 2.3 already used it to describe explicitly the compressed c.i. algebras when $r=2$. We shall need below only a weak corollary where each degree $d_{i}$ is 0 , and the $B_{i}$ are constants.

Lemma 3.1 (Classical JoRdan Lemma-SeE [16, APPendix 3]). Suppose $L_{1}, \ldots, L_{n}$ are distinct linear forms (none is a multiple of another) in $\mathscr{R}=k\left[y_{1}, y_{2}\right]$; and that the integers $d_{1}, \ldots, d_{n}$ satisfy $\Sigma\left(d_{i}+1\right) \leqslant j+1$. Then there is no nontrivial relation $\sum B_{i} L_{i}^{j-d_{i}}=0$, with coefficients $B_{i} \in \mathscr{R}_{d_{i}}$. In particular, taking $d_{i}=0$, we see that any $j+1$ perfect $j$ th powers of distinct linear forms are linearly independent, hence span $\mathscr{R}_{j}$.

Corollary 3.2 (Powers of LiNEAR FORMS SPAN ALl FORMS (Classical)). Given $s$ and a vector subspace $V$ of $\mathscr{R}_{s}$ (in $r$ variables) there are $\left(\# \mathscr{R}_{s}-\# V\right)$ perfect sth powers of linear forms spanning a space complementary to $V$ in $\mathscr{R}_{s}$.

Proof. It suffices to show that $M_{s}$, the span of $s$ th powers of linear forms in $\mathscr{R}_{1}$, is in fact all $\mathscr{R}_{s}$. Since $\mathscr{R}_{1}=M_{1}$, it suffices to show $\mathscr{R}_{1} M_{s}=M_{s+1}$, or that $B, L \in \mathscr{R}_{1}$ implies $B L^{s} \in M_{s+1}$. The latter inclusion follows formally from the case $r=2$ in Lemma 3.1.

The following proposition is the basic existence result we need. The special case $V=0$ shows that compressed Gorenstein algebras of expected maximal length exist (Theorem I). The proof uses Corollary 3.2 twice, and the properties of Zariski open sets.

Proposition 3.3A. (INDEPENDENCE OF HIGHER DERIVATES OF A FORM mod A GIVEN SPACE). Suppose the integers $s, j$ with $j / 2 \leqslant s \leqslant j$ are given, and that the subspace $V$ of $\mathscr{R}_{s}$ satisfies $\# V \leqslant\left(\# \mathscr{R}_{s}-\# \mathscr{R}_{j-s}\right)$. Then there is a form of degree $j$ such that the vector space $(J F)_{s}=J^{j-s} F$ of $(j-s)$ th partial derivatives of $F$ satisfies both

$$
\#(J F)_{s}=\# \mathscr{R}_{j-s} \text { and }(J F)_{s} \cap V=0 \text {. }
$$

The set of all such forms $F$ (up to nonzero constant multiple) satisfying (2) comprises an open dense subset of the projective space $\mathbf{P}\left(\mathscr{R}_{j}\right)$.

Proposition 3.3B (SURJECTIVITY: HIGHER DERIVATES OF A FORM SPAN $\mathscr{R}_{s} \bmod V$ ). If $s \leqslant j / 2$, there are forms $F$ with $(J F)_{s}=\mathscr{R}_{s}$. If $s \leqslant j$, there are $F$ with $\#(J F)_{s}=$ $\min \left(\# \mathscr{R}_{s}, \# \mathscr{R}_{j-s}\right)$; and if also $\# V \geqslant \# \mathscr{R}_{s}-\# \mathscr{R}_{j-s}$ there are $F$ with $\left((J F)_{s}+V\right)$ $=\mathscr{R}_{s}$. The set of all forms $F$ (up to nonzero constant multiple) satisfying any of these conditions is parametrized by an open dense subset of $\mathbf{P}\left(\mathscr{R}_{j}\right)$. 
Proof of Proposition 3.3A. Let $n=\# \mathscr{R}_{j-s}$. Since $j-s \leqslant s$, the integer $n=$ $\# \mathscr{R}_{j-s} \leqslant \# \mathscr{R}_{s}$. Consider $n$ linear forms $L_{1}, \ldots, L_{n}$ with $L_{i}=b_{i 1} y_{1}+\cdots+b_{i r} y_{r}$, and let $B=\left(b_{i j}\right) \in k^{n r}$. As $n \leqslant \# \mathscr{R}_{s}$, Corollary 3.2 implies that for a choice of the matrix $B$ in a Zariski open dense subset $U_{V}$ of $\mathbf{A}^{n r}$, the vector space $L^{(s)}$ $=\left\langle L_{1}^{s}, \ldots, L_{n}^{s}\right\rangle$ satisfies both $\# L^{(s)}=n=\# \mathscr{R}_{j-s}$ and $\left(L^{(s)} \cap V=0\right)$. Note that the condition of missing $V$ is open on $\mathbf{A}^{n r}$ and is nonempty by Corollary 3.2. To complete the proof, we need only show there is a form $F \in \mathscr{R}_{j}$ with $(J F)_{s}=L^{(s)}$. Consider $F=L_{1}^{j}+\cdots+L_{n}^{j}$. Note that if $u$ denotes $j-s$, and $D$ the multi-index $D=\left(d_{1}, \ldots, d_{r}\right)$ of length $u$, then the partial derivative $\left(\partial^{u} / \partial y^{D}\right)\left(L_{i}^{j}\right)=$ $(j ! / s !)\left(L_{i}^{s}\right)\left(B_{i}\right)^{D}$, where $\left(B_{i}\right)^{D}=\left(b_{i 1}^{d_{1}}\right) \cdots\left(b_{i r}^{d_{r}}\right)$. Thus, taking each partial as a row, and each $L^{s}$ as a column, the matrix expressing $J^{j-s} F$ in terms of an independent set of powers $L_{1}^{s}, \ldots, L_{n}^{s}$ is $(j ! / s !) M$, where:

$$
M=\left(\begin{array}{lll}
L_{1}^{s} & & L_{n}^{s} \\
b_{11}^{u} & & b_{n 1}^{u} \\
b_{11}^{u-1} b_{12} & & b_{n 1}^{u-1} b_{n 2} \\
\cdots \cdots & \ldots \ldots \cdots & \cdots \\
b_{1 r}^{u} & & b_{n r}^{u}
\end{array}\right) \begin{aligned}
& \partial^{u} / \partial y_{1}^{u} \\
& \partial^{u} / \partial y_{1}^{u-1} \partial y_{2} \\
& \partial^{u} / \partial y_{r}^{u}
\end{aligned}
$$

In other words $(j ! / s !) M\left(L_{1}^{s}, \ldots, L_{n}^{s}\right)^{T}=\left(\partial^{u} F / \partial y_{1}^{u}, \ldots, \partial^{u} F / \partial y_{r}^{u}\right)^{T}$, where $T$ denotes transpose. The matrix $M$ also satisfies $\left(L_{1}^{u}, \ldots, L_{n}^{u}\right)=\left(y_{1}^{u}, y_{1}^{u-1} y_{2}, \ldots, y_{r}^{u}\right) D M$, where $D$ is the diagonal matrix having as principle diagonal the multinomial coefficients

$$
\left(\begin{array}{l}
u \\
u
\end{array}\right),\left(\begin{array}{c}
u \\
u-1,1
\end{array}\right), \ldots,\left(\begin{array}{l}
u \\
u
\end{array}\right) .
$$

Corollary 3.2 now applied to $\mathscr{R}_{u}$ shows that $\operatorname{det} M \neq 0$ for $B$ in a Zariski open set $U^{\prime}$ of $\mathbf{A}^{n r}$. Thus, for $B$ in the Zariski-open dense subset $U_{V} \cap U^{\prime}$ of affine space $\mathbf{A}^{n r}$, the space $L^{(s)}$ of $s$ th powers has size $\# L^{(s)}=n$, misses the original vector space $V$, and satisfies $J^{j-s} F=L^{(s)}$. This completes the proof of Proposition 3.3A.

Proof of Proposition 3.3B. For the first statement, if $s \leqslant j / 2$ choose $n=\# \mathscr{R}_{s}$ and show $(J F)_{s}=\mathscr{R}_{s}$ using the method of 3.3A. The middle statement is now already shown. If $\# V \leqslant \# \mathscr{R}_{s}-\# \mathscr{R}_{j-s}$ and $j / 2 \leqslant s$, choose a subspace $V^{\prime}$ of $V$ satisfying $\# V^{\prime}=\# \mathscr{R}_{s}-\# \mathscr{R}_{j-s}$; we conclude from Proposition 3.3A that there are degree-j forms $F$ with $(J F)_{s} \cap V^{\prime}=0$ but $\#(J F)_{s}=\# \mathscr{R}_{j-s}$, hence that $\left((J F)_{s}+\right.$ $\left.V^{\prime}\right)=\mathscr{R}_{s}$.

The following proposition shows that graded compressed algebras of socle type $E$ and expected length $n(E)$ exist, and, in fact, are common (Theorem I). The degrees $D$ of a set $S$ of forms determine the degree-type $E(S)$, as $e_{u}=\#\left\{i \mid d_{i}=u\right\}$. Let $R_{-1}=R_{-2}=\cdots=0$. Recall that the polynomial $P \in R=k[[X]]$ acts on $F \in \mathscr{R}$ as a higher partial derivate $P(F)$ (see Definitions 1 or $2.3 \mathrm{ff}$.). Note, $\# R_{S}=\# \mathscr{R}_{S}$.

PROPOSITION 3.4 (INDEPENDENCE OF HIGHER DERIVATES OF A SET OF FORMS). If $F_{1}, \ldots, F_{e}$ are general enough forms of degrees $D=\left(d_{1}, \ldots, d_{e}\right)$ in $\mathscr{R}$, then for each $s$ the vector space homomorphism $h=h(s): R_{d_{1}-s}+\cdots+R_{d_{e}-s} \rightarrow \mathscr{R}_{s}: h\left(P_{1}, \ldots, P_{e}\right)$ $=\sum P_{i}\left(F_{i}\right)$ is either injective or surjective according to whether $\left(\sum \# R_{d_{i}-s} \leqslant \# R_{s}\right)$ or $\left(\sum \# R_{d_{i}-s} \geqslant \# R_{s}\right)$. 
Proof. Assume $d_{1} \geqslant \cdots \geqslant d_{e}$ and choose first $F_{1}$ so that each space $\left(J F_{1}\right)_{s}$ satisfies $\#\left(\left(J F_{1}\right)_{s}\right)=\min \left(\# R_{s}, \# R_{d_{1}-s}\right)$. Proposition 3.3B implies that for each $s$, the $F_{1}$ satisfying this condition are parametrized by an open dense subset $U(1, s)$ of the projective space $\mathbf{P}\left(\mathscr{R}_{d_{1}}\right)$. The intersection $U(1)=\cap U(1, s)$ is also open dense, since $\mathbf{P}\left(\mathscr{R}_{d_{1}}\right)$ is an irreducible variety, and $U(1)$ parametrizes all the forms $F_{1}$ satisfying the condition on \# $\left(\left(J F_{1}\right)_{s}\right)$ for each $s$. For each $s$ the restriction $h_{1}(s)=h(s) \mid R_{d_{1}-s}$ is injective or surjective to $\mathscr{R}_{s}$ according to whether $\# R_{d_{1}-s} \leqslant$ $\# R_{s}$, or $\# R_{d_{1}-s} \geqslant \# R_{s}$. Suppose, by way of induction, that open dense sets $U(i) \subset \mathbf{P}\left(\mathscr{R}_{d_{i}}\right)$ have been chosen for $i=1, \ldots, u-1$ in such a way that if each $F_{i}$ is parametrized by a point of $U(i)$, then for each $s$ the restriction map $h_{u-1}(s)=$ $h(s) \mid\left(R_{d_{1}-s}+\cdots+R_{d_{(u-1)}-s}\right) \rightarrow \mathscr{R}_{s}$ is injective or surjective, according to whether $\Sigma_{1}^{u-1} \# R_{s-d_{i}} \leqslant \# R_{s}$ or the opposite. For each $s$, Proposition 3.3B with $j=d_{u}$ and $V=\left(J\left(F_{1}, \ldots, F_{u-1}\right)\right)_{s}$ yields an open dense set $U(u, s)$ in $\mathbf{P}\left(\mathscr{R}_{d_{u}}\right)$ parametrizing the forms $F_{u}$ for which the next restriction $h_{u}(s)$ is injective or surjective to $\mathscr{R}_{s}$, according to whether $\sum_{1}^{u} \# R_{d_{1}-s} \leqslant \# R_{s}$ or the opposite. Let $U(u)=\bigcap_{s} U(u, s)$, an open dense set in $\mathbf{P}\left(\mathscr{R}_{d_{u}}\right)$. This completes the induction step, and the proof.

PROPOSITION 3.5 (INDEPENDENCE OF HIGHER DERIVATES OF A SET OF FORMS mOd GIVEN SPACES). Under the hypotheses of Proposition 3.4, if also for each $s$ the subspace $W_{s}$ is fixed in $\mathscr{R}_{s}$, the induced homomorphism $\bar{h}(s)=\pi \circ h(s)$ to $\mathscr{R}_{s} / W_{s}$ is for general $F_{1}, \ldots, F_{e}$ an injection or surjection according to whether $\left(\left(\sum \# R_{d_{i}-s}\right)+\left(\# W_{s}\right)\right) \leqslant \# R_{s}$ or the opposite.

Proof. Similar to that of Proposition 3.4 but taking in the induction step $V=\left(J\left(F_{1}, \ldots, F_{u-1}\right)\right)_{s}+W_{s}$.

It is easy to see from Proposition 3.5 that, given an Artin algebra $A=R / I$ having dual module $W \subset \mathscr{R}$, and a socle-type $E$ permissible relative to $A$, one can construct new algebras $A(S)$ compressed relative to $A$, whose dual modules $V^{\prime \prime}=W+J S$ have the maximal length $n(A ; E)$ specified in Definition 2.5. This shows the existence part of Theorem III.

3B. Parametrizing sets of polynomials having the most derivates. This key section describes the family $Z(E)$ of subspaces $W=J S$ of the polynomial ring $\mathscr{R}=k[Y]$ having maximal length, given the degree-type $E=H(S)$ of the generators $S$ of $W$. We start with the family $G(E)$ parametrizing graded subspaces $W$, then handle the general case. Proposition 3.7, showing that $Z(E)$ is a bundle over $G(E)$ with affine space fibers, results from an improvement over the methods of [18]; the other results extend the methods there from one socle generator to many. These results complete the proofs of Theorems I-III of §2D. Since this section is more technical than the preceding one, the reader may wish to look first at some applications of these results in $\S 4 \mathrm{~A}$.

We begin with the graded case. Recall that $S_{>i}$ denotes either a graded module $S$ having nonzero pieces only in degree greater than $i$, or the degree greater-than- $i$ part of a graded module $S$. The sequence $E_{\geqslant i}$ is $\left(0, \ldots, 0, e_{i}, e_{i+1}, \ldots\right)$, the tail of $E$. We parametrize families of length $n$ vector subspaces $V_{S}$ in $R$ having no elements of degree greater than $j$, via the reduced subscheme structure inherited from the 
Grassmannian Grass $\left(n, J \mathscr{R}_{j}\right)$ parametrizing $n$-dimensional subspaces of $J \mathscr{R}_{j}=\mathscr{R}_{0}$ $\oplus \cdots \oplus \mathscr{R}_{j}$. The notation $J S_{>i}$ means $J\left(S_{>i}\right)$.

Proposition 3.6 (Graded compressed modules relative to $V$ ). Suppose the graded J-module $V$ is given, as well as a sequence $E$ permissible as socle type, satisfying $e_{i} \neq 0 \Rightarrow\left(h_{i}(E)+h_{i}(V)\right) \leqslant \# R_{1}$ (see Definition 2.5). Let the sequence $H=$ $H(V ; E)=H(V)+H(E)$ in the sense of Definition 2.4, and let

$$
n(V ; E)=\# H(V ; E)=h_{0}+h_{1}+\cdots .
$$

Then there is a nonempty variety $G(V ; E)$ parametrizing graded modules $V_{S}=V+J S$ of length $n(V ; E)$, generated relative to $V$ by subspaces $S$ in $\mathscr{R}$ of degree-type $E$. There is a natural projection $G\left(V, E_{\geqslant i}\right)$ onto $G\left(V ; E_{>i}\right)$. Its fiber $F_{p}$ over a point $p \in$ $G\left(V ; E_{>i}\right)$ parametrizing the graded module $\left(V+J S_{>i}\right)$ is the open dense subset $F_{p}$ in the Grassmannian Grass $\left(e_{i}, R_{i} /\left(V+J S_{>i}\right)\right)$ parametrizing classes of $e_{i}$-dimensional subspaces $S_{i}$ in $\mathscr{R}_{i} \bmod \left(\left(V+J S_{>i}\right) \cap \mathscr{R}_{i}\right)$ for which the length of $\left(V+J S_{>i}+J S_{i}\right)$ is $n\left(V ; E_{\geqslant i}\right)$, the maximum possible value. The variety $G(V ; E)$ is irreducible, and is covered by opens in an affine space of dimension $g(V ; E)=\sum\left(\# R_{i}-h_{i}\right)\left(e_{i}\right)$.

Proof. Beginning with a large $j, G\left(V ; E_{\geqslant j}\right)=G(V ; 0)$ is a point. The existence of an $S_{i}$ satisfying \# $\left(V+J S_{\geqslant i}+J S_{i}\right)=n\left(V ; E_{\geqslant i}\right)$ results from repeated application of Proposition 3.5. There is a natural embedding of an open dense subset of $\operatorname{Grass}\left(e_{i}, \mathscr{R}_{i} /\left(\left(V+J S_{>i}\right) \cap \mathscr{R}_{i}\right)\right)$ parametrizing such $S_{i}$ into $\operatorname{Grass}\left(n\left(V ; E_{\geqslant i}\right), J \mathscr{R}_{j}\right)$, defined by polynomial equations, arising from the map $S_{i} \rightarrow\left(V+J S_{>i}+J S_{i}\right)$. (This also proves Proposition 1A.)

It remains to verify the local structure. Consider the standard basis $B=$ $\left\{1, y_{1}, \ldots, y_{r}^{j}\right\}$ for $J \mathscr{R}_{j}$. Choose an open $U(W)$ in $\operatorname{Grass}\left(n\left(V ; E_{>i}\right), J \mathscr{R}_{j}\right)$, defined by the choice of a subset $W$ of $B$ having $\left(\#\left(J \mathscr{R}_{j}\right)-n\left(V ; E_{>i}\right)\right.$ ) elements as complementary basis to $V$. Since the $V+J S_{>i}$ are graded, we may assume that $W_{i}=W \cap \mathscr{R}_{i}$ has $\left(\# R_{i}-\#\left(\left(V+J S_{>i}\right) \cap \mathscr{R}_{i}\right)\right)$ elements. Then for each point of $U(W)$ the different spaces $S_{i}$ may be chosen in the fixed space $\left\langle W_{i}\right\rangle$, hence there is an immersion $G\left(V ; E_{\geqslant i}\right) \mid U(W)$ onto an open dense set of $\operatorname{Grass}\left(e_{i},\left\langle W_{i}\right\rangle\right) \times U(W)$. The image is open-dense, as each fibre $G\left(V ; E_{\geqslant i}\right)$ over $U(W)$ is open dense in $\operatorname{Grass}\left(e_{i},\left\langle W_{i}\right\rangle\right)$. Thus, $G(V ; E)$ is locally an affine space, of dimension

$$
\sum \operatorname{dim}\left(\operatorname{Grass}\left(e_{i},\left\langle W_{i}\right\rangle\right)\right)=\sum\left(\# W_{i}-e_{i}\right)\left(e_{i}\right)=\sum\left(\# R_{i}-h_{i}(V ; E)\right)\left(e_{i}\right)
$$

as claimed.

The following proposition shows that a compressed algebra has compressed associated graded algebra (see Corollary 3.8). The key step below is (IV) $\Leftrightarrow$ (V). Given the space $V$ and a degree-type $E$, let $t$ be the largest integer for which $h_{t}(V ; E)=\# R_{t}$, in the notation of Proposition 3.6; the integer $t$ also satisfies $t=v(I)-1$ where $v(I)$ is the initial degree of ideal $I=\operatorname{Ann}(V+J S)$ defined by a relatively compressed module $V+J S$ where $S$ has degree-type $E$ (Proposition 3.5). Recall that $J \mathscr{R}_{t}=\mathscr{R}_{0} \oplus \cdots \oplus \mathscr{R}_{t}$. To state (IV) and (V) we need to consider the image of $R$ acting on $S$, mod $\left(J \mathscr{R}_{t}+V\right)$. Given $\left(f_{1}, \ldots, f_{e}\right)$ of degree-type $E$ in $\mathscr{R}$ with $e_{u}$ of degree $u$ let $d_{i}=\operatorname{degree} f_{i}$, let the module $D_{i}=R_{0} \oplus \cdots \oplus R_{d_{i}-(t+1)}$, 
and denote by $D$ the exterior direct sum $D=D_{1} \oplus \cdots \oplus D_{e}$. The homomorphism $\tau: D \rightarrow \mathscr{R} /\left(J \mathscr{R}_{t}+V\right)$ is defined by

$$
\tau\left(p_{1}, \ldots, p_{e}\right)=p_{1}\left(f_{1}\right)+\cdots+p_{e}\left(f_{e}\right) \bmod \left(J \mathscr{R}_{t}+V\right) .
$$

Note that the image of $\tau$ is $\left(J S+\left(J \mathscr{R}_{t}+V\right)\right) /\left(J \mathscr{R}_{t}+V\right)$. We define $\tau^{*}$ similarly, using the initial forms $\left(F_{1}, \ldots, F_{e}\right)$ in place of $\left(f_{1}, \ldots, f_{e}\right)$ and the graded module $V^{*}$ in place of $V$.

Proposition 3.7 (COMPARISON OF GENERAL AND GRADED COMPRESSED MODULES). Suppose that $V$ is a J-closed submodule of $R$, and that $V^{*}=\mathrm{Gr} V$ is its associated graded module $V^{*}=\oplus V_{i}$, as defined in (1) of $\$ 2 \mathrm{~A}(\mathrm{~d})$ or at the start of $\S 3 \mathrm{~A}$. Assume that $E$ is a sequence permissible relative to $V$ in the sense of Proposition 3.6, that $S=\left(f_{1}, \ldots, f_{e}\right)$ is a subspace of $\mathscr{R}$ having generator type equal to its degree-type $E$, and that $e_{u}$ of the $f$ 's have degree $u$, for each $u$. Let the space of top-degree forms of $f_{1}, \ldots, f_{e}$ be $S^{*}=\left(F_{1}, \ldots, F_{e}\right)$, also of degree-type $E$. Then the following are equivalent:

(I) The space $J S+V$ has maximal dimension $n(V ; E)$ among spaces $J S^{\prime}+V$, with $S^{\prime}$ of degree-type $E$.

(II) The space $J S^{*}+V^{*}$ has maximal dimension $n(V ; E)$ among the spaces $\left(J S^{\prime}+V^{*}\right)$ with $S^{\prime}$ graded of degree-type $E$.

(III) The space $(J S+V)$ has the maximal degree-type $H(V ; E)=H(V)+$ $\sum e_{i} H(i)$. (See Definition 2.4 of $\$ 2 \mathrm{D}$ and Proposition 3.6.)

(IV) The homomorphism $\tau$ is an injection, and $(J S+V) \supset J \mathscr{R}_{t}$.

(V) The homomorphism $\tau^{*}$ is an injection and $\left(J S^{*}+V^{*}\right) \supset J \mathscr{R}_{t}$.

Proof. The sequence of proof is first (II) $\Leftrightarrow$ (V) $\Rightarrow$ (IV), then (I) $\Leftrightarrow$ (IV) $\Leftrightarrow$ (III), and finally the key part (IV) $\Rightarrow(\mathrm{V})$. Note that $S^{*}$ also has degree-type $E$, since $J_{1} S \cap S=0$. First, Proposition 3.6 shows there are graded spaces $S^{\prime}$ of degree-type $E$ yielding the maximal size \# $\left(J S^{\prime}+V^{*}\right)=n(V ; E)$. It is easy to see that for such a space $S^{\prime}$ the homomorphism $\tau^{*}$ is an injection and $\left(J S^{\prime}+V^{*}\right) \supset J \mathscr{R}_{t}$. Furthermore, the integer $n(V ; E)$ is greater than or equal to the length $\#(J S+V)$ for any space of $S$ of degree-type $E$ : This is true since the monomial partial derivates of $f_{i}$ excluded from the space $D_{i}$ all map $f_{i}$ to $J \mathscr{R}_{t} \subset\left(J S^{\prime}+V^{*}\right)$. Thus, the length $\#(J S+V) \leqslant$ (\#(image $\tau)+\#\left(J \mathscr{R}_{t}+V\right)$ ); the length is maximal when $\tau$ is injective and $J S+V$ $\supset J \mathscr{R}_{t}$. Thus (II) $\Leftrightarrow(\mathrm{V})$. Since (V) $\Rightarrow$ (IV) is immediate, the maximal length $n(V ; E)$ is in fact attained by some \#(JS+V), hence (I) $\Leftrightarrow$ (IV). It is easy to verify that (IV) $\Leftrightarrow$ (III). To complete the proof, we need to show (IV) $\Rightarrow(V)$, especially when $V$ is not graded, that is when $V \neq V^{*}$.

Suppose that the space $S$ of degree-type $E$ satisfies $\#(J S+V)=n(V ; E)$, and suppose by way of contradiction that $\tau^{*}$ is not injective. Then $\sum p_{i}\left(F_{i}\right)=0 \bmod V^{*}$, with $p_{i} \in D_{i}$ and $F_{i}$ the leading (top degree) form of $f_{i}$. Since $F_{i}$ and $V^{*}$ are graded we may suppose each $p_{i}$ is a form and that each $p_{i}\left(F_{i}\right)$ has the same degree $u>t$. Choose a form $P$ in $R_{u-(t+1)}$. Then

$$
0=P\left(\sum p_{i}\left(F_{i}\right)\right)=\sum P p_{i}\left(F_{i}\right) \bmod J \mathscr{R}_{t}=\sum P p_{i}\left(f_{i}\right) \bmod J \mathscr{R}_{t}
$$

(since each $P p_{i}\left(f_{i}\right)$ has degree $\left.t+1\right)$, contradicting the injectivity of $\tau$. Hence $\tau$ injective implies $\tau^{*}$ injective. 


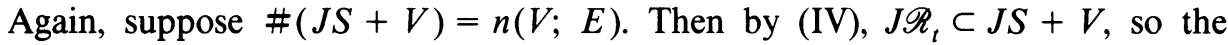
quotient space $\mathscr{R}_{t}$ satisfies

$$
\begin{aligned}
\mathscr{R}_{t} & =J \mathscr{R}_{t} / J \mathscr{R}_{t-1}=\left((J S+V) \cap J \mathscr{R}_{t}\right) / J \mathscr{R}_{t-1} \\
& =\left(\left(J S^{*}+V_{t}\right) \cap J \mathscr{R}_{t}\right) / J \mathscr{R}_{t-1}
\end{aligned}
$$

by the injectivity of $\tau$ : no partial derivates of order lower than expected land in $J \mathscr{R}_{t}$. Consequently $J S^{*}+V^{*}$ includes $J \mathscr{R}_{t} \bmod J \mathscr{R}_{t-1}$, which is only possible if $J S^{*}+V^{*}$ includes $J \mathscr{R}_{t}$. This completes the proof of $(\mathrm{IV}) \Rightarrow(\mathrm{V})$, and of the proposition.

Corollary 3.8 (The algebra $A$ IS COMPRESSED IfF $A^{*}$ IS COMPRESSED). The compressed algebra $A$ whose dual module is generated by $f_{1}, \ldots, f_{e}$ has a compressed associated graded algebra $A^{*}$ whose dual module is generated by $F_{1}, \ldots, F_{e}$, the top-degree forms of $f_{1}, \ldots, f_{e}$. Conversely, $A^{*}$ compressed implies $A$ is compressed; then the socle type $E(A)=E\left(A^{*}\right)$.

The following proposition is the important step in showing the parametrization parts of Theorems II and III. The case where $V=0$ appears in [18] as Lemma 3.33A.

Proposition 3.9 (FAMILIES OF MODUles that ARE THE SUM OF $V$ AND A COMPRESSED SIMPLE MODULE). Suppose the vector subspace $V \subset \mathscr{R}$ is $j$-closed, of degree-type $H(V)$. Suppose $j$ is an integer satisfying $j \geqslant \min \left(i \mid h_{i}(V) \neq \# R_{i}\right)$. Let $H=H(V ; j)=(H(V)+H(j))=\left(h_{0}, h_{1}, \ldots\right)$ as in Definition 2.4 , let $n(V ; j)=\sum h_{i}$ $=\sum h_{i}(V ; j)$ and let $t+1=\min \left(i \mid h_{i}(V ; j) \neq \# R_{i}\right)$. Let $f=\left(f_{j}+f_{j-1}+\cdots+f_{t}\right)$ be a polynomial in $\mathscr{R}$ of degree $j$, and $V^{\prime}=V_{f}$ the module $V^{\prime}=V+J f$. Then the variety $Z(V ; j)$ parametrizing the modules $V_{f}$ of maximal length $n(V ; j)$ is an affine space $\mathbf{A}^{N}$ bundle over the variety $G(V ; j)$ parametrizing those modules $V_{F}$ of maximal length where $F$ is a form $F=f_{j}$. The variety $Z(V ; j)$ is rational, irreducible, and has dimension $\sum_{i \leqslant j}\left(\# R_{i}-h_{i}\right)$. The variety $G(V ; j) \cong G\left(V^{*} ; j\right)$, and is isomorphic to an open dense subset of the projective space $\mathbf{P}\left(\mathscr{R}_{j} / V_{j}\right)$.

Proof. First, Proposition 3.7 shows that $V_{f}$ has maximal length if $V_{F}^{*}$ does, with $F=f_{j}$. This and Proposition 3.6 show that $G(V ; j) \cong G\left(V^{*} ; j\right)$, and each is parametrized by an open dense subset in $\mathbf{P}\left(\mathscr{R}_{j} / V_{j}\right)$. To prove the assertion about $Z(V ; j)$ we must parametrize the distinct modules $V_{f}$ of maximal length. Since two modules $V_{f}$ and $V_{f^{\prime}}$ can differ only by those elements of $\left(J f+J f^{\prime}\right)$ having degree at most $j$, it suffices to parametrize the distinct $\bar{V}_{f}=V_{f} \cap J \mathscr{R}_{j}$. Thus $Z(V ; j)$ is naturally a subvariety, with reduced scheme structure, of the Grassmannian $\operatorname{Grass}\left(n^{\prime}, J \mathscr{R}_{j}\right)$ parametrizing length $n^{\prime}$ quotients of $J \mathscr{R}_{j}$, where $n^{\prime}=\sum_{i \leqslant j} h_{i}^{\prime}$. Suppose that the degree-j form $F$ is fixed, with $V_{F}$ of maximal length, and choose vector spaces $W_{i}$ so that $W_{i}$ is a direct-sum complement in $\mathscr{R}_{i}$ to $(V F)_{i}$, for each $i<j$ : thus $\left(W_{i}+(J F)_{i}+V_{i}\right)=\mathscr{R}_{i}$ for each $i<j$ where $W_{i} \neq 0$. We need

LEMMA 3.10 (STANDARD GENERATOR FOR $V_{f}$ ). For each module $M=V_{f}$, where the leading form $f_{j}=F$, there is a unique choice of a polynomial $f(M)=F+f_{j-1}+$ $\cdots+f_{t+1}$ with each $f_{i} \in W_{i}$ for $i<j$, for which $M=V_{f(M)}$. 
Proof of Lemma 3.10. First we choose $f(M)$, then we will show it is unique. Given such a module $M=V_{f}$, suppose $u<j$ is the integer such that the components $f_{u+1}, \ldots, f_{j-1}$ are in $W_{u+1}, \ldots, W_{j-1}$ respectively, but $f_{u} \notin W_{u}$. (If $f_{j-1} \notin W_{j-1}$ then $u=j-1$.) Since $W_{u}$ is a complement to $(J F)_{u}+V_{u}$ in $\mathscr{R}_{u}$, the element $f_{u}=w_{u}+v_{u}$ $+h(F)$, where $w_{u} \in W_{u}, v_{u} \in V_{u}$, and $h(F) \in \mathscr{R}_{u}$ (so $h=h(F)$ is some $(j-u)$ th partial derivative of $F)$. Choose $f^{\prime}=((1-h) \circ(f)-v)$ where $v \in V$ is any element having top degree form $v_{u}$. Then $f_{i}^{\prime}=f_{i}$ for $i>u$; and $f_{u}^{\prime}=\left(f_{u}-h(F)-v_{u}\right)=w_{u}$ $\in W_{u}$. The module $V_{f^{\prime}}=J f^{\prime}+V=J((1-h) \circ f)+V=J f+V=M$, since $(1-$ $h)$ has inverse $\left(1+h+\cdots+h^{j}\right)$ in its action as partial differential operator on $J f$. Thus, we have improved $f$ to an element $f^{\prime}$ of $\mathscr{R}$ such that $(f-F)$ belongs to $W_{i}$ for $i \geqslant u$. Continuing in this way, we find a generator $f(M)$ with $(f(M)-F) \in W=$ $\oplus W_{i}$.

If the polynomials $f$ and $f^{\prime}$ both satisfy the condition (if both $(f-F) \in W$, and $\left.\left(f^{\prime}-F\right) \in W\right)$ and $V_{f}=V_{f^{\prime}}=M$, suppose that $G=\left(f-f^{\prime}\right)_{u}$ in $W_{u}$ is the top degree form of $\left(f-f^{\prime}\right)$. Then $\left(f-f^{\prime}\right) \in M$ implies also $G \in M_{u}=(J F)_{u}+V_{u}$; the equality follows from Proposition 3.7 and uses the assumption that $V_{f}$ has maximal length $n(V ; j)$. But the inclusion $G \in M_{u}$ contradicts the choice of $W_{u}$ complementary to $J F+V_{u}$; thus, $f=f^{\prime}$. This completes the proof of the uniqueness of $f(M)$, and of the lemma.

Proof of Proposition 3.9, CONTINUED. Lemma 3.10 shows that the fibre of $Z(V ; j)$ over the point in $G(V ; j)$ parametrizing $V_{F}$ is the affine space $\mathbf{A}^{N}$ built on the vector space $W=\oplus W_{i}$. The fibre dimension is $N=\# W=\sum_{i<j}\left(\# R_{i}-h_{i}\right)$, so $z(V ; j)=\operatorname{dim} Z(V ; j)=\sum_{i \leqslant j}\left(\# R_{i}-h_{i}\right)$, since the variety $G\left(V^{*} ; j\right)$ parametrizing the forms $F$ is an open in $\mathbf{P}\left(\mathscr{R}_{j} / V_{j}\right)$. To verify that $Z(V ; j)$ is a locally trivial bundle over $G(V ; j)$, note that if a choice of vector spaces $W_{i}$ (of right size, missing $V_{i}$ ) is made first (before $F$ ), they are complementary to $V_{F}^{*}$ for forms $F$ parametrized by an open subset $U_{W}$ of $\mathbf{P}\left(\mathscr{R}_{j} / V_{j}\right)$. It is easy to see that over $U_{W}$ the bundle $Z(V ; j)$ is trivial: a point of the fibre $\mathbf{A}^{N}$ corresponds to a choice of set of forms $f_{i} \in W_{i}$, one for each $i \leqslant j-1$. Over each point $z_{F} \in U_{W}$ corresponding to a module $V_{F}^{*}$, this choice of each $f_{i}$ determines a polynomial $f=F+\sum f_{i}$ and a unique compressed module $M_{f}$, whose associated graded module $M_{f}^{*}=M_{f}=V_{f}^{*}$. The algebraicity of the map $\mathbf{A}^{N} \times U_{W} \rightarrow Z(V ; j)$ completes the proof of local triviality and of Proposition 3.9.

Proposition 3.11 (FAmily of Relatively COMPRESSED modules). Suppose the vector subspace $V$ of $\mathscr{R}$ is $J$-closed, and that the sequence $E$ is permissible for the degree-type of the space $S$ in $A_{S}$, in the sense of Definition 2.5A using there $A=R /$ Ann $V$, or $H(A)=H(V)$. Then the variety $Z(V ; E)$ parametrizing maximal length $R$-modules $V_{S}=J S+V$, with $S$ of degree-type $E$, is a locally trivial bundle over $G(V ; E)$ parametrizing those maximal length modules $V_{S}$ where $S$ is graded of degree-type $E$. The fibre of $Z(V ; E)$ over $G(V ; E)$ is an affine space $\mathrm{A}^{N}$ of dimension $N=\Sigma_{i} \Sigma_{u>i}\left(e_{u}\right)\left(\# R_{i}-h_{i}(V ; E)\right)$. Hence $Z(V ; E)$ is an irreducible variety of dimension $z(V ; E)=\sum_{i} \sum_{u \geqslant i}\left(e_{u}\right)\left(\# R_{i}-h_{i}(V ; E)\right)$, that is locally an affine space. 
Proof. Immediate from Propositions 3.6, 3.7, and the method of proof of Proposition 3.9. Note that when $V$ is not graded, the modules $V_{S}$ parametrized by $G(V ; E)$ need not be graded.

Proof of Theorems I-III ( $\$ 2 \mathrm{D})$. The remaining parametrization parts of these theorems are now consequences of Propositions 3.9, and 3.11, together with the duality of $\S 2$.

\section{Applications, comments.}

4A. Nonsmoothable algebras. This section begins with some examples of compressed algebras, to illustrate the Hilbert function and dimension calculations. Among these examples are some families of algebras whose general member is not smoothable. Such a nonsmoothable algebra has no deformation to the trivial algebra $k \oplus \cdots \oplus k$.

EXAMPLE 4.1. Smoothable Gorenstein algebra. When $r=4$ and $E=(0,0,0,1,0, \ldots)$ of type 1 , the Hilbert function $H(E)$ is $(1,4,4,1)$ of length 10 . The variety $G(E)$ parametrizing compressed graded Gorenstein algebra quotients of $R$ having socle degree 3 is an open subvariety of $\mathbf{P}\left(R_{3}\right)=\mathbf{P}_{19}$. To find the dimension of the variety $Z(E)$ subtract $(1,4,4,1)$ termwise from the sequence $\left(\# R_{0}, \# R_{1}, \ldots, \# R_{3}\right)$, namely $(1,4,10,20)$ obtaining $(0,0,5,19)$; then $z(E)=\operatorname{dim} Z(E)=5+19=24$, and the fibre of $Z(E)$ over $G(E)$ is $\mathbf{A}^{5}$. R. Buchweitz has shown that the family consists of smoothable algebras.

Note. Generic Gorenstein algebras. R. Buchweitz pointed out in 1978 that there are errors in the hand-calculated matrix $M$ that appears on p. 170 of [18]. The correct $M$ has smaller rank. The matrix $M$ is constructed using repeated blocks, so an error is repeated. As he remarks, when $A$ is Gorenstein, the dimension \# $\operatorname{Hom}(I, A)=$ $\#\left(I / I^{2}\right)$, which is readily seen to be at least $(6+19+(35-21))=39$ here. A more recent computer calculation of $\#\left(I / I^{2}\right)$ in fact yields ranks $(6+19+15)=40$ in general, consistent with smoothability. Thus, the claim of $\$ 3.2$ of [18] for Gorenstein algebras $A$ with $H(A)=(1,4,4,1)$ is incorrect: the small tangent space method for producing generic algebras does not apply there. The recent computer calculations strongly suggest that $H(A)=(1,5,5,1)$ Gorensteins are smoothable. They also suggest that general enough $H(A)=(1,6,6,1)$ Gorenstein algebras are the first $(1, r, r, 1)$ case where the small tangent space method of [18] would apply, to yield "generic" Gorenstein algebras having deformations only to other algebras of the same kind. Verification awaits a confirming calculation (the program yields the expected dimensions of $I / I^{2}$ in 6 variables, and "self-confirms" its validity in 4, 5 variables, but errs in 7 variables).

EXAMPLE 4.2. Likely smoothable. When $r=4$ and $E=(0,0,0,2)$, the Hilbert function $H(E)=(1,4,4,1)+(1,4,4,1)=(1,4,8,2)$ of length 15 . The variety $G(E)$ is a dense open in the Grassmannian Grass $\left(2, R_{3}\right)$, and has dimension $g(E)=$ $2\left(\# R_{3}-2\right)=2(20-2)=36$. The bundle $Z(E)$ over $G(E)$ has fibre an affine space of dimension $2\left(\# R_{2}-8\right)=2(10-8)=4$, so $\operatorname{dim} Z(E)=2(2+18)=40$.

EXAMPLE 4.3. Nonsmoothable compressed algebras in 3 variables. When $r=3$ and $E=(0,0,0,0,0,0,2,5)$ is the socle type of algebras having 5 dual generators of 
degree 7 and 2 of degree 6 , the Hilbert function

$$
\begin{aligned}
H(E) & =2(H(6))+5(H(7))=2(1,3,6,10,6,3,1)+5(1,3,6,10,10,6,3,1) \\
& =(1,3,6,10,15,21,17,5)
\end{aligned}
$$

of length $n(E)=78$. The variety $Z(E)$ has dimension $7(28-17)+5(36-5)=232$ and is a bundle over $G(E)$, which has dimension $2(28-17)+5(36-5)=177$. The variety $Z(E)$ parametrizes algebra quotients of $R$, which can be regarded as algebras concentrated at the origin of affine 3-space $\mathbf{A}^{3}$. Moving $Z(E)$ forms a bundle $Z(E)^{\prime} \subset \operatorname{Hilb}^{78}\left(\mathbf{A}^{3}\right)$ over $\mathbf{A}^{3}$ having dimension $\operatorname{dim}\left(Z(E)^{\prime}\right)=235$. The variety $U(3,78)$ parametrizing sets of 78 distinct points of $\mathbf{A}^{3}$ has dimension only $3(78)=234$. Thus, the general compressed Artin algebra of this socle type $E$ is not smoothable: $Z(E)^{\prime} \not \subset \overline{U(3,78)}$. This is the smallest length nonsmoothable algebra known to the author when $r=3$; there is every reason to expect examples of smaller length, not detectable by a dimension method.

EXAMPLE 4.4. Nonsmoothable type 2 algebra in 3 variable. When $r=3$, and there are only two dual generators of degree 11, then the Hilbert function $H(E)=$ $(1,3,6,10,15,21,28,30,20,12,6,2)$ of length 112 . Then $\operatorname{dim} Z(E)=420$, yielding a bundle $Z(E)^{\prime}$ over $\mathbf{A}^{3}$ of dimension 423. $\operatorname{But} \operatorname{dim}(U(3,112))=3(112)=336$, much smaller. This shows that the general compressed algebra $A$ with 2 degree-11 dual-generators is not smoothable. Since Kleppe has shown that Gorenstein Artin algebras of embedding dimension 3 are smoothable, type 2 is the smallest type of nonsmoothables when $r=3$. Since type 2 algebras deform only to type 2 or type 1 algebras, and these nonsmoothables by [25] cannot deform to type 1, one may conclude that there is a component of some $\mathrm{Hilb}^{n} R$, with $n \leqslant 112$, parametrizing generic type 2 algebras having deformations only to other algebras of the same kind (see [20]).

EXAMPLE 4.5. $A$ smoothable Gorenstein algebra $A$ that is not alignable. When $r=3$ and there is one socle basis element of degree 7 , then $H(E)$ is $(1,3,6,10,10,6,3,1)$ of length 40 , and $\operatorname{dim} Z(E)=80$. The dimension of the variety parametrizing aligned algebra quotients $A^{\prime}$ of $R$ (where $A^{\prime}=k[x] /\left(x^{40}\right)$ ) is $(3-1)(40-1)=78$. These Gorenstein algebras are smoothable by [25], but cannot be deformed to $A^{\prime}$ - they are not alignable. This example, generalized in Theorem 3.35 of [18] was the first known example of such behavior. It is now known that most complete intersections of embedding dimension at least three are smoothable but not alignable (see [14, 22, and 23]).

Nonsmoothable Artin algebras have also been studied in [19 and 28]. In [29] dimension methods are used to produce nonsmoothable families of curves; Buchweitz has shown how to use nonsmoothable Artin algebras to produce "very rigid" higher dimension algebras, rings of the miniversal deformation space of the nonsmoothable algebra [11, §5.2.10].

4B. Almost linear resolutions (with D. Buchsbaum and D. Eisenbud). This section resumes joint work with D. Buchsbaum and D. Eisenbud, whom I thank for their comments, and their permission to include this summary. We show that graded 
compressed algebras of certain socle types $E$ have almost linear resolutions. D. Buchsbaum and D. Eisenbud have in certain cases determined the maps that occur in the resolution [10].

Definition 4.1. The minimal free resolution $\mathscr{B}$ of a graded algebra $A=R / I$ is almost linear if the maps $\phi_{2}, \ldots, \phi_{r-1}$ each have degree 1 , in

$$
\mathscr{B}: 0 \stackrel{\phi_{r}}{\rightarrow} B_{r} \rightarrow \cdots \rightarrow B_{2} \stackrel{\phi_{2}}{\rightarrow} B \stackrel{\phi_{1}}{\rightarrow} \stackrel{R}{\|}_{0} \stackrel{\phi_{0}}{\rightarrow} A=R / I \rightarrow 0 .
$$

EXAmple 4.6. A graded Gorenstein algebra $A$ having Hilbert function $H(A)=$ $(1,3,1)$ has resolution

$$
0 \rightarrow R(-5) \rightarrow R^{5}(-3) \rightarrow R^{5}(-2) \rightarrow R \rightarrow A \rightarrow 0
$$

which is almost-linear.

The following proposition was shown in the special case that $A$ is Gorenstein by R. Stanley [18, footnote, p. 179], and independently by P. Schenzel (Theorem B of [34], case $\operatorname{dim} R=0$ ). It was extended by D. Buchsbaum, D. Eisenbud, and the author to the general case.

Proposition 4.1A (GRADED COMPRESSED ALGEBRAS WITH ALMOST LINEAR RESOLUTION). The graded compressed algebra $A=R / I$ of socle type $E$ has almost-linear resolution if $E$ or $A$, respectively, satisfies (TFAE):

(i) $\sum e_{i}\left(\# R_{i-(v-1)}\right)=\# R_{v-1}$, where $v$ is the initial degree of $I$.

(ii) There are no nontrivial differential relations $\sum P_{i}\left(F_{i}\right)=0$ satisfying each $P_{i}$ a form in $R$ and $\left(\operatorname{deg} F_{i}-\operatorname{deg} P_{i}\right)=v-1$, among the generators $\left(F_{1}, \ldots, F_{e}\right)$ of the dual module $\hat{A} \subset \mathscr{R}$ for A.

Proposition 4.1B. The degrees of the maps and the ranks of the modules $B_{i}$ for these algebras $A$ depend only on the socle-type $E$ satisfying (3). The module $B_{i}$ is generated by elements of degree $(v+i-1)$, for $1 \leqslant i<r$; the ideal $I$ (and $\left.B_{1}\right)$ is generated entirely in degree $v$.

Proof that A(i) $\Leftrightarrow \mathrm{A}(\mathrm{ii})$. Let $d_{i}$ be the degree of $F_{i}$, and consider the homomorphism of Proposition 3.4, $h: \oplus R_{d_{i}-s} \rightarrow \mathscr{R}_{s}, h\left(P_{1}, \ldots, P_{e}\right)=\sum P_{i}\left(F_{i}\right)$, for the degree $s=v-1$. The length of the domain of $h$ is $\sum \# R_{d_{i}-(v-1)}=\sum e_{i}\left(\# R_{i-(v-1)}\right)$, and the length of $\mathscr{R}_{v-1}$ is $\# R_{v-1}$. Since $A$ is compressed, $h$ has maximal rank; hence $\mathrm{A}(\mathrm{i}) \Rightarrow h$ is an isomorphism $\Rightarrow h$ is injective $\Leftrightarrow \mathrm{A}(\mathrm{ii})$. Conversely, $\mathrm{A}(\mathrm{ii}) \Leftrightarrow h$ injective and maximal rank $\Rightarrow \#($ domain $h) \leqslant \# \mathscr{R}_{v-1}$. Since the ideal $I$ has initial degree $v$, $I_{v-1}=0$ and $h$ must be onto $\mathscr{R}_{v-1}$ : by $\S 2 \mathrm{~A}$, the colength (image $h$ ) in $\mathscr{R}_{v-1}$ equals $\# I_{v-1}$. Hence $h$ is an isomorphism. Thus $\mathrm{A}(\mathrm{ii}) \Rightarrow \mathrm{A}(\mathrm{i})$. 
Proof of Propositions 4.1A(i) and 4.1B. Let $u=\left(\left(\sum e_{i}\left(\# R_{i-(v-2)}\right)\right)-\# R_{v-2}\right)$ and $d=\# I_{d}=\# R_{v}-\left(\sum e_{i}\left(\# R_{i-v}\right)\right)$. Then the minimal resolution of the algebra $A$ satisfying the condition $\mathrm{A}(\mathrm{i})$, begins and ends

$$
\begin{array}{cccc} 
& B_{r} & & B_{r-1} \\
0 \rightarrow & \oplus R^{e_{i}}(-i-r) & \stackrel{\phi_{r}}{\rightarrow} & \left(R^{u}(-r-(v-2))+\text { higher }\right) \cdots \\
& B_{1} & & B_{0} \\
\stackrel{\phi_{2}}{\rightarrow} & \left(R^{d}(-v)+\text { lower }\right) & \stackrel{\phi_{1}}{\rightarrow} & R \rightarrow A .
\end{array}
$$

Here -1 is higher than -2 . Each map $\phi_{i}$ splits into parts $\phi_{i j}=\left(\phi_{i} \mid j\right.$ th summand of $B_{i}$ ) having degree at least 1 . In a string of such component maps, the degrees of the pieces of $B_{r-1}, \ldots, B_{1}$ that are involved must increase by at least one at each step, hence by $(r-2)$ from $B_{r-1}$ to $B_{1}$. This does not permit higher terms in $B_{r-1}$ nor lower terms in $B_{1}$ than $R^{u}(-r-(v-2))$, or $R^{d}(-v)$, respectively. It follows that each $B_{i}$ for $1<i<r-1$ has summands of degree $(-i-(v-1))$ only, implying the resolution is almost linear. This completes the proof of 4.1A, and of 4.1B.

Example 4.7 (R. Stanley, P. Schenzel). Compressed Gorenstein algebras of even socle degree. These satisfy for all $r$ the condition A(i) so they have almost linear resolutions. A specific example is a compressed algebra $A$ of Hilbert function $H(A)=(1,3,6,3,1)$ having one dual generator of degree 4; its resolution "skeleton" is $0 \rightarrow R^{1}(-7) \rightarrow R^{7}(-3) \rightarrow R^{7}(-2) \rightarrow R \rightarrow A$. See Example 2.4.

EXAMPLE 4.8. A compressed algebra $A$ satisfying $H(A)=(1,3,6,1)$ and $E(A)=$ $(0,0,3,1)$ is defined by an ideal $I$ of initial degree 4 ; but the first differential relations happen in degree 2 - there are none in degree $3=4-1$. Thus, $A$ satisfies $\mathrm{A}(\mathrm{ii})$, and has almost linear resolution $0 \rightarrow R(-6)+R^{3}(-5) \rightarrow R^{12}(-4) \rightarrow$ $R^{9}(-3) \rightarrow R \rightarrow A$.

EXAMPLE 4.9. Algebras $A=R /(V)$ where $V$ is a large space of degree-j forms. If $A$ is compressed, if $I$ is generated by $d$ forms $V=\left(F_{1}, \ldots, F_{d}\right)$ all of degree $j$ in $R$, if $d$ is large enough so the integer $w=\left(\# R_{j-1}-r\left(\# R_{j}-d\right)\right)$ is nonnegative, then the socle-type $E(A)=\left(0, \ldots, 0, w, \# R_{j}-d, 0, \ldots\right)$, the Hilbert function $H(A)=$ $\left(1, \ldots, \# R_{j-1}, \# R_{j}-d, 0, \ldots\right)$ and $A$ has almost-linear resolution. When $r=3$, $j=4$, and $d=12$, the integer $w=10-3(15-12)=1$ is nonnegative; thus compressed algebras of socle type $E(A)=(0,0,0,1,3,0)$ and Hilbert function $H(A)=$ $(1,3,6,10,3,0)$ have almost linear resolutions. In the language of $\$ 4 \mathrm{C}$ to follow, the algebras of this example are both compressed and thin.

Note. Constructing socle types of almost linear compressed algebras. Example 4.9 illustrates the process. Fix the initial degree $v(i)$. Choose $e_{i}$ for $i \geqslant v$ so that the integer $w=\# R_{v-1}-\sum_{i \geqslant v} e_{i}\left(\# R_{i-(v-1)}\right)$ is nonnegative, and add on $w$ generators for $\hat{A}$ of degree $v-1$.

4C. Ideals generated by general polynomials - thin algebras. Another way to form an extreme class of local algebras $B=R / I$ - the thin algebras-is to specify the orders of the generators of the defining ideal $I$ in the ring $R=k\left[\left[x_{1}, \ldots, x_{r}\right]\right]$, then 
insist that each graded piece $I_{i}=\left(I \cap m^{i}+m^{i+1}\right) / m^{i+1}$ be as large as possible. Here we say $f \in R$ has order $i$, or initial degree $i$ if $m^{i} \supset f$ but $m^{i+1} \not \supset f$.

Definition 4.2A. The generator type of an ideal $I$ in $R$ is the sequence $D=$ $\left(d_{1}, \ldots, d_{i}, \ldots\right)$ where $d_{i}=\# V_{i}$, the length of the degree- $i$ piece

$$
V_{i}=\left(V \cap m^{i}+m^{i+1}\right) / m^{i+1}
$$

of a minimal generating set $V$ for $I$. The integer $d_{i}$ satisfies

$$
d_{i}=\left(\#\left(\left(I \cap m^{i}\right) /\left(m\left(I \cap m^{i-1}\right)\right)\right)-\#\left(\left(I \cap m^{i+1}\right) /\left(m\left(I \cap m^{i}\right)\right)\right)\right) .
$$

Intuitively, $d_{i}$ is the number of generators of $I$-rightly counted-having order $i$. We denote the total number of generators of $I$ by $\mu(I)=\sum d_{i}$.

Definition 4.2B. Fix $r$. An algebra quotient $B=R / I$ is thin if it has the smallest possible length $\# B=\operatorname{dim}_{k} B$ among the algebra quotients of $R$ defined by ideals of generator type $D$. Here we assume also that the sequence $D$ is permissible for the generator type of a thin algebra: if $d_{i}>0$ then no algebra quotient $B^{\prime}$ of $R$, defined by an ideal having generator type $D_{<i}=\left(d_{0}, \ldots, d_{i-1}, 0, \ldots\right)$ satisfies $\# B_{i}^{\prime}<d_{i}$.

For a general enough subset $S$ of $R$, having $d_{i}$ elements of each order $i$, the quotient algebra $B=R /(S)$ will be thin of type $D$. We outline below what is known about thin algebras.

First, thin is quite distinct from compressed-a compressed algebra $A$ has general dual generators; this often causes the defining ideal $I$ of $A$ to be very special among ideals of the same generator type. Thus, when $r>2$ the only compressed c.i. algebras are those having socle degree 1 or Hilbert function $H=(1,3,3,1)$ (and are thin); but the c.i. algebra $B(N)=R /\left(x_{1}^{n_{1}}, \ldots, x_{r}^{n_{r}}\right)$ is thin.

EXAMPLE 4.10. Thin vs. compressed algebras. The algebra $B=R /\left(x_{1}^{3}, x_{2}^{3}, x_{3}^{3}\right)$ is a thin complete intersection algebra having Hilbert function $H(B)=(1,3,6,7,6,3,1)$. A compressed Gorenstein algebra $A$ having the same socle degree 6 as $B$ has Hilbert function $H(A)=(1,3,6,10,6,3,1)$; the defining ideal $I$ of $A$ has 9 generators all of degree 4 , but they satisfy special relations-the algebra $A$ is not thin. Any thin algebra $B^{\prime}$ defined by an ideal having 9 sufficiently general generators of degree 4 , has Hilbert function $H\left(B^{\prime}\right)=(1,3,6,10,6,0)$ and is also compressed of socle type $(0,0,0,0,6)$.

EXAMPLE 4.11. The simplest thin algebras when $r=2$. Suppose $V \subset R_{j}$ is a general vector space of degree- $j$ forms, of fixed size $\# V=n$. Then when $r=2$ the space $R_{s} V=\left(\left\{f g \mid f \in R_{s}, \quad g \in V\right\}\right)$ satisfies $\# R_{s} V=\min \left(\left(\# R_{s}\right)(\# V), \# R_{s+j}\right)$. The spaces $V$ satisfying this condition for each $s$ define thin algebras $A=R /(V)$, which are Artin algebras when $\# V>1$.

The following proposition characterizes compressed algebras and thin algebras $A=R / I$ when $r=2$. Recall that when $r=2$ and $A$ is an Artin algebra the Hilbert function $H=H(A)$ satisfies $H=\left(1,2, \ldots, d, h_{d}, \ldots, h_{j}, 0\right)$ with $d \geqslant h_{d} \geqslant h_{d+1} \geqslant$ $\cdots \geqslant h_{j}>0$, where $j$ is the highest socle degree of $A$, and $d$ is the initial degree of the ideal $I$. Theorem 4.3 of [21] implies that the minimum possible number of generators for the defining ideal $I$ of $A$ is $\mu(I)=\left(1+\max \left(\Delta_{i}\right)\right)$, where the difference $\Delta_{i}=h_{i-1}-h_{i}$. The proof of Theorem 4.3, and Theorem 2.12 of [21] show 
that the minimum is attained for a Zariski-dense subset of the family of all algebras, quotients of $R$, having Hilbert function $H(A)$.

Proposition 4.2A (Generator tyPE AND Hilbert FUnCTION; THIN Algebras). If $r=2$, and the ideal I has generator type $D$, the Hilbert function of the Artin algebra $B=R / I$ satisfies the inequality

$$
\Delta_{i} \leqslant\left(\sum_{u \leqslant i} d_{u}\right)-1 .
$$

The algebra $B$ is thin iff there is equality in (4) for each $i$ for which $h_{i} \neq 0$. Equivalently, an algebra $B$ is thin iff $H(B)$ satisfies $\Delta_{d} \leqslant \Delta_{d+1} \leqslant \cdots \leqslant \Delta_{j}$, and the ideal I has the minimum possible number of generators $\mu(I)=\left(1+\max \left(\Delta_{j}, \Delta_{j+1}\right)\right)$. Then I has $\Delta_{d}+1$ generators of degree $d$, and $\left(\Delta_{i}-\Delta_{i-1}\right)$ generators of degree $i$, for $i=d+1, \ldots, j, j+1$. Furthermore, when $r=2$, the algebra $B$ is thin iff its associated graded algebra $B^{*}$ is thin. Thus, the thin algebras of generator type $D$ form an affine space bundle of fibre $\mathbf{A}^{N}, N=\sum_{u<i} d_{u} h_{i}$, over the family of graded thin algebras of generator type $D$; the latter form an open subvariety of the irreducible variety $G_{H}$ of dimension $\sum d_{i} h_{i}$ parametrizing graded algebras of the Hilbert function $H$ satisfying equality in (4).

Proposition 4.2B (SOCle TyPe AND Hilbert FUnCTION: COMPRESSED ALGEBRAS). If $r=2$, any algebra $A$ of socle type $E$ has Hilbert function satisfying

$$
\Delta_{i} \leqslant \sum_{u \geqslant i-1} e_{u}
$$

The algebra $A$ is compressed iff there is equality for each $i$ for which $h_{i-1} \neq i$. Equivalently, an algebra $A$ is compressed iff $H(A)$ satisfies $\Delta_{d+1} \geqslant \Delta_{d+2} \geqslant \cdots \geqslant$ $\Delta_{j+1}$, and the ideal $I$ has the minimum possible number of generators $\mu(I)=(1+$ $\left.\max \left(\Delta_{d}, \Delta_{d+1}\right)\right)$.

Proof of Proposition 4.2A. Fix a generating set $L=\left(f_{1}, \ldots, f_{\mu}\right)$ for $I$ having $d_{i}$ elements of order $i$. Let the ideal $I(s)=(\{f \in I \mid \operatorname{order} f \leqslant s\})$ be generated by the lower-order elements of $L$. Note that the Hilbert function $H(I(s))$ agrees with $H(I)$ in degrees no larger than $s$. Theorem 4.3 of [21] applied to $R / I(s)$ shows that the differences $\Delta_{i}^{\prime}$ for $H(R / I(s))$ satisfy $\Delta_{i}^{\prime} \leqslant\left(\sum_{u \leqslant s} d_{u}\right)-1$. Since $\Delta_{s}=\Delta_{s}^{\prime}$ this shows the inequality (4) for $i=s$. This argument actually requires an elementary extension of Theorem 4.3 of [21] to quotients $A^{\prime}=R / I(s)=R / I^{\prime}$ that may not be Artinian: then $I^{\prime}=f I^{\prime \prime}$ where $A^{\prime \prime}=R / I^{\prime \prime}$ is Artinian, and $f$ is a polynomial; applying Theorem 4.3 to $A^{\prime \prime}$ gives the extension to $A^{\prime}$. This completes the proof of the inequality (4). Evidently, the length $\# B=\sum h_{i}$ is minimum when the differences are maximum: for the length $\# B$ equals

$$
\left(1+\cdots+d+\left(d-\Delta_{d}\right)+\left(d-\Delta_{d}-\Delta_{d+1}\right)+\cdots+\left(d-\Delta_{d}-\cdots-\Delta_{j}\right)\right) \text {. }
$$

Again, Theorem 4.3 and 2.12 of [21] show there are algebras $B$ having Hilbert function $H(B)$ satisfying equality in (4), and that a Zariski dense subset of them have $\mu(I)=\sum d_{i}$ generators, hence these are defined by ideals $I$ of generator type $D$. The equivalent condition stated is simply a numerical translation of equality in (4). 
We now show that when $r=2, B$ is thin iff $B^{*}$ is thin. Suppose $B=R / I$ is thin, and let $\left(f_{d}, \ldots, f_{0}\right)$ be standard generators for $I$ in a weak-normal pattern for $B$ [21, §4A]. Recall that order $f_{d} \leqslant \operatorname{order} f_{d-1} \leqslant \cdots \leqslant \operatorname{order} f_{0}$, and that $f_{d}, \ldots, f_{0}$ is not usually a minimal set of generators. Suppose $M$ is the relation matrix for the $f$ 's, whose $i$ th row is $\left(\alpha_{i, 0}, \ldots, \alpha_{i, i-1}-y, \ldots, \alpha_{i, d}\right)$, and let $M^{\prime}$ be $M \bmod (x, y)$, so $M^{\prime}$ has entries the constant terms $\alpha_{u, v}^{\prime}$ of $\alpha_{u, v} \in k[x]$. Suppose $\Delta_{t-1}$ and $\Delta_{t}$ are nonzero. Then for some $i, f_{i+\Delta_{t-1}}-1, \ldots, f_{i}$ have order $t-1$ and $f_{i-1}, \ldots, f_{i-\Delta_{t}}$ have order $t$. Since $\alpha_{u, v}^{\prime}=0$ when order $f_{v} \leqslant \operatorname{order} f_{u-1}, M^{\prime}$ is possibly nonzero on, to the left of, and below the $\Delta_{t-1} \times \Delta_{t}$ block $\left\{\alpha_{u, v}^{\prime}\right.$ with $i+1 \leqslant u \leqslant i+\Delta_{t-1}$ and $i-\Delta_{t} \leqslant v \leqslant i$ -1 \}, but $M^{\prime}$ is 0 to the right of or above the block. If $B$ is thin, then $I \bmod m^{t+1}$ in $R \bmod m^{t+1}$ has $1+\Delta_{t}$ generators if $t \leqslant j$, or $1+\max \left(\Delta_{j}, \Delta_{j+1}\right)$ generators if $t=j+1$; this fact results from equality in (4) and the restriction that the sequence $D$ is permissible for a generator type of a thin algebra (Definition 4.2B). However, the minimum number of generators for $I \bmod m^{t+1}$ in $R / m^{t+1}$ is $\left(\# f_{i}\right.$ of order $\left.\leqslant t\right)$ - (rank of the corresponding columns of $\left.M^{\prime}\right)=\left(1+\Delta_{d}+\cdots+\Delta_{t}\right)$ - (rank of the last $1+\Delta_{d}+\cdots+\Delta_{t}$ columns of $M^{\prime}$ ). Thus, for $B$ to be thin, the rank of these last columns must be $\left(\Delta_{d}+\cdots+\Delta_{t-1}\right)$. Since $\Delta_{d} \leqslant \Delta_{d+1} \leqslant \cdots \leqslant \Delta_{j}$, this is only possible for each $t$ beginning with $d+1$ if each $\Delta_{t-1} \times \Delta_{t}$ block of $M^{\prime}$ described above has maximal rank $\Delta_{t-1}$. These blocks for $M^{\prime}$ are the same as those for the corresponding matrix $M^{\prime \prime}$ for $B^{*}$ : for $M^{\prime \prime}$ differs from $M^{\prime}$ only in having its entries below or left of the blocks all zero. Thus, $B$ thin implies $B^{*}$ is thin when $r=2$. The converse is immediate.

The remaining statements are immediate from Theorems $2.11,2.12$ and 3.14 of [21].

Proof of Proposition 4.2B. Fix a generating set $S$ for the dual module of $A$ in $R$. Let $A(s)=R / I[s]$ be the algebra whose dual module is generated by $(\{f \in$ $S \mid$ degree $f \geqslant s\})$. Then the Hilbert function $H(A(s))$ with differences $\Delta_{i}^{\prime}$ agrees with $H(A)$ in degrees no smaller than $s$. When $r=2$, the number of generators of $I$ and the type of $A$ are related by $\mu(I)=e(A)+1$. Thus, applying Theorem 4.3 of [21] to $A(s)$, we find

$$
e(A(s))+1=\left(\left(\sum_{i \geqslant s} e_{i}\right)+1\right)=\mu(I[s]) \geqslant \max \left(\Delta_{i}^{\prime}\right)+1 .
$$

Since $\Delta_{s+1}=\Delta_{s+1}^{\prime}$ we have shown the inequality (5) for $i=s+1$.

The Hilbert function of a compressed algebra of socle type $E$ is $H(E)=\sum e_{i} H(i)$ by Theorem IIA. When there is equality in (5), the Hilbert function of $A$ is evidently $H(E)$; this shows that $A$ is compressed iff there is equality in (5). The equivalent condition is a numerical translation using Theorem 4.3.

Combining the criteria of Propositions 4.2A and 4.2B, we determine the overlap. The notation $a^{+}$denotes $\max (0, a)$.

CoRollary 4.3 (Algebras Both THIN AND COMPRESSED). If $r=2$, the algebra $A=R / I$ is both thin and compressed iff $H(A)$ satisfies $\Delta_{d} \leqslant \Delta_{d+1}=\cdots=\Delta_{j} \geqslant$ $\Delta_{j+1}$, and I has the minimum possible number of generators $\mu(I)=1+\Delta_{d+1}$. (In the case $d=j, H(A)=\left(1, \ldots, d, d-\Delta_{d}, 0\right)$.) The ideal I has $\left(\Delta_{d}+1\right)$ generators of 
degree $d$, and $\left(\Delta_{d+1}-\Delta_{d}\right)^{+}$of degree $d+1$, and no others; also, the algebra $A$ has the socle type $E=\left(0, \ldots, 0, e_{j-1}, e_{j}\right)$ with $e_{j}=\Delta_{j+1}$ and $e_{j-1}=\left(\Delta_{j}-\Delta_{j+1}\right)^{+}$. Thus when $r=2, A$ is thin and compressed iff $A$ is thin and $I$ is generated either in one degree or in adjacent degrees-or equivalently if $A$ is compressed and has socle generators in one or in adjacent degrees.

Now consider $r \geqslant 3$. The Hilbert function of a thin complete intersection Artin algebra is that of some algebra $B(N)=R /\left(x_{1}^{n_{1}}, \ldots, x_{r}^{n_{r}}\right)$, where $N$ denotes $\left(n_{1}, \ldots, n_{r}\right)$. Such thin c.i. Artin algebras have thin c.i. associated graded algebras; a straightforward parametrization of them is in [23, Lemma 3 and Note 1].

In the next case, $\mu(I)=r+1$, where $A$ is an almost complete intersection Artin algebra, R. Stanley has shown that the Hilbert function $H(A)$ is the one expected. Suppose $n_{1} \leqslant \cdots \leqslant n_{r} \leqslant n_{r+1}$, and $N=\left(n_{1}, \ldots, n_{r}\right)$. Assume now that char $k=0$.

Proposition ((R. Stanley) A thin almost complete intersection algebra $B$ DEFINED BY AN IDEAL $I$ OF GENERATOR ORDERS). $N^{\prime}=n_{1}, \ldots, n_{r+1}$ has Hilbert function $H(B)=H\left(B^{\prime}\right)$ where $B^{\prime}=B(N) /\left(\sigma^{n_{r+1}}\right)$ and $\sigma$ is a general linear combination of $x_{1}, \ldots, x_{r}$. Here $H\left(B^{\prime}\right)$ can be determined by the property that the homomorphism multiplication by $\sigma^{n_{r+1}}$, mapping $B(N)_{i}$ to $B(N)_{i+n_{r+1}}$, is injective for all integers satisfying $i \leqslant\left(\left(\sum_{1}^{r} n_{u}\right)-n_{r+1}\right) / 2$, and is surjective for larger $i$.

Proof. The ring $B(N)$ is the cohomology ring of a smooth product $X=\prod \mathbf{P}^{n_{i}-1}$ of projective spaces. The conclusion about injectivity and surjectivity of $\sigma^{n_{r+1}}$ acting on $B(N)$ is an aspect of the Hard Lefschetz theorem on $X$. The Koszul relations require that any algebra $B$ defined by an ideal $I$ of generator orders $n_{1}, \ldots, n_{r+1}$ either has greater length than $B^{\prime}$, or is thin and has Hilbert function $H(B)$ equal to $H\left(B^{\prime}\right)$.

Since the quotient ring $B^{\prime}$ is not the cohomology ring of a smooth manifold, there is no immediate extension of Stanley's proof to the more general case where $\mu(I) \geqslant r+2$. Recently, D. Anick has used combinatorial methods to characterize the Hilbert functions of thin algebras when $r$ is three [36]. The only other general result known to the author is the following proposition due to $M$. Hochster, communicated by D. Laksov. When a thin algebra is defined by an ideal $I$ having generators all of the same degree $d$, the proposition determines $h_{d+1}$.

Proposition ((M. Hochster) Linear MUltiples of $V$ ). If $V \subset R_{d}$ is a general enough vector space of forms of fixed dimension $\# V$, then the space $R_{1} V$ has the expected dimension, $\# R_{1} V=\min \left(r(\# V), \# R_{d+1}\right)$, provided $d>1$.

The proof involves considering the natural map $R_{1}^{(\# V)} \times R_{d}^{(\# V)} \rightarrow R_{d+1}$. In degree 2 , the case $V$ spanned by monomials is a combinatorial problem solved by C. Lech.

We do not even know when $r>2$ if $A$ thin implies that the associated graded algebra $A^{*}$ is also thin! (See Proposition 4.2 for the case $r=2$.)

D. Laksov has proposed the problem, given a general $d$-dimensional vector space of forms of degree $j$, to compute the rank of the homomorphism $\theta: R_{s+j-u} \rightarrow$ $\operatorname{Hom}\left(R_{u}, R_{s+j} / R_{s} V\right)$ defined by $\theta(f) \circ(g)=f g \bmod R_{s} V$. He derives the answer in 
the case $R_{1} \rightarrow \operatorname{Hom}\left(R_{j}, R_{1+j} / R_{1} V\right)$ from the proposition just shown. The general problem is equivalent to determining the size of the kernel

$$
K(\theta)=R_{-u} R_{s} V=\left\langle\left\{f \in R_{s+j-u} \mid R_{u} f \subset R_{s} V\right\}\right\rangle .
$$

I know of no further results when $r>2$. In the case of two variables this problem is readily solved using a convenient measure of the complexity of the space $V$, namely $\tau(V)=\# R_{1} V-\# V=\# V-\#\left(R_{-1} V\right)$, a number satisfying $\tau\left(R_{1} V\right) \leqslant$ $\tau(V)$, and $\tau\left(R_{-1} V\right) \leqslant \tau(V)$ (see [21, 24]). The principle is that beginning with a general length- $d$ space $V$ in $R_{j}$, any sequence of spaces, each related in a fixed way to the preceding, such as $V, R_{1} V, R_{2} V, R_{-1} R_{2} V, R_{-2} R_{2} V, R_{1} R_{-2} R_{2} V$, has the property that the nonincreasing sequence of integers $\tau(V), \tau\left(R_{1} V\right), \ldots$ is as constant as possible, and drops only when forced by space limitations. Even when $d\left(\# R_{s}\right)<$ $\# R_{j+s}$ (so that $R_{s} V$ is not $R_{j+s}$ ), there can be a space limitation in degree $(s+j+1)$ forcing $\tau\left(R_{s} V\right)$ to be less than $\tau(V)$, and affecting the expected size of $R_{-u} R_{s} V$, the kernel of the homomorphism $\tau$. This occurs in Case B of Proposition 4.4. Note that $s>0$, and, for all $i$, \#R $=i+1$. The phrase "most spaces" as usual refers to those parametrized by a Zariski-open subset of the Grassmannian $\operatorname{Grass}\left(d, R_{j}\right)$.

Proposition 4.4. Euclidean algorithm: expected size of $R_{-u} R_{s} V$ when $r=2$.

Case A (No space limitation). If $d\left(\# R_{s+1}\right) \leqslant\left(\# R_{s+j+1}\right)$, then for most length-d subspaces $V$ of $R_{j}$, the space $R_{-s} R_{s} V$ is $V$, and for each $u \geqslant 0$, the space $R_{-u} R_{s} V$ is $R_{s-u} V$. If $u \leqslant s$, then $\# R_{s-u} V=d\left(\# R_{s-u}\right)$; otherwise $R_{s-u} V=0$.

Case B (Space limitation in degree $s+j+1)$. Assume that $d\left(E R_{s+1}\right)>\# R_{s+j+1}$, but that $d\left(\# R_{s}\right)<\# R_{s+j}$. Then for most length-d subspaces $V$ of $R_{j}$, the integer $d=\tau(V)=\cdots=\tau\left(R_{s-1} V\right)$ but $R_{s+1} V=R_{s+j+1}$; hence, the integer $\tau^{\prime}=\tau\left(R_{s} V\right)$ equals $\# R_{s+j+1}-\#\left(R_{s} V\right)=\# R_{s+j+1}-d\left(\# R_{s}\right)$ which is less than $d$. So $R_{s} V$ is simpler than $V$. For general $V$ and for each $u \geqslant 0$, the codimension in $R_{s+j-u}$ of $R_{-u} R_{s} V$ is $\min \left((u+1)\left(\tau^{\prime}-1\right), \# R_{s+j-u}\right)$, and the dimension $\#\left(R_{-u} R_{s} V\right)=$ $\left(\# R_{s+j+1}-(u+1) \tau^{\prime}\right)^{+}$.

Case C (Space limitations in degree $s+j)$. If $d\left(\# R_{s}\right) \leqslant \# R_{s+j}$, then for general length-d spaces $V$ in $R_{j}$, the space $R_{s} V=R_{s+j}$ and $R_{-u} R_{s} V=R_{s+j-u}$ for all $u$.

Proof. The simplest Case $\mathrm{C}$ results from choosing a thin algebra of generator type $(0, \ldots, 0, d, 0, \ldots)$ and using Proposition 4.2A. The Case A results from taking $\tau^{\prime}=d$ in Case B. To show Case B, let $A=R / I$ be a compressed algebra of socle type $E=\left(0, \ldots, 0, d-\tau^{\prime}, \tau^{\prime}-1,0, \ldots\right)$ where $e_{s+j}=\tau^{\prime}-1$. By Corollary 4.3 the algebra $A$ is compressed and thin, and $\mu(I)=e+1=d$. Also, by Theorem II, $h_{j}=\operatorname{cod} I_{j}=(s+1)\left(\tau^{\prime}-1\right)+s\left(d-\tau^{\prime}\right)$ where $\tau^{\prime}=s+j+2-d(s+1)$. Thus $h_{j}=j+1-d$, and a similar calculation shows $h_{j-1}=j$. The space $V=I_{j}$ satisfies $\# V=d$, and $I_{j-1}=0$; these facts with $v(I)=d$ imply that the ideal $I$ is generated by $V$, hence $I_{s+j}=R_{s} V$. But we may choose the algebra $A$ so that the algebra $A^{\prime}=R / I^{\prime}$, defined by $I^{\prime}=\operatorname{Ann}\left(\left(I_{s+j}\right)^{\perp} \cap \mathscr{R}_{s+j}\right)$ and having socle type $E^{\prime}=$ $\left(0, \ldots, 0, \tau^{\prime}-1,0, \ldots\right)$, is also compressed. Here $I^{\prime}$ is the ancestor ideal of $I_{s+j}$ as in 
[24]. Its graded piece $I_{s+j-u}^{\prime}=R_{-u} I_{s+j}=R_{-u} R_{s} V$ and has codimension in $R_{s+j-u}$, $h_{s+j-u}^{\prime}=\min \left((u+1)\left(\tau^{\prime}-1\right), \# R_{s+j-u}\right)$, as claimed. It is easy to see that there is a Zariski open family of length- $d$ spaces $V$ in $R_{j}$ for which $A=R /(V)$ is thin and compressed, and for which $A^{\prime}$ is also compressed. This completes the proof.

The sequence of subspaces $V, R_{-1} V, R_{-2} R_{2} V, \ldots$, in $R_{j}$ has limit $(f) \cap R_{j}$ where $f$ is the greatest common divisor of elements of $V$; the sequence performs the steps in the Euclidian algorithm. This is, of course, essentially one variable algebra, done in a proper homogeneous setting. The principle of constancy of $\tau$ for a sequence of related spaces is more general than the proposition above, which illustrates it (see [24]).

When $r \geqslant 3$, the work of G. Gotzmann, concerning spaces $R_{s} V$, where the length $\# R_{1} V$ is minimum, has a relation to the size of $R_{-u} R_{s} V$ for $s$ large (see [13, and 22, Appendix]). The work of D. Berman [5, 6], and of D. Bayer [4] also is relevant.

4D. Closures of $Z(E)$ and $G(E)$; power sum algebras of socle type $E$. The varieties $Z(E)$ and $G(E)$, parametrizing compressed algebras and graded compressed algebras of socle type $E$, have closures $\overline{Z(E)}$ and $\overline{G(E)}$ in the Hilbert scheme Hilb ${ }^{n} R$ parametrizing all length- $n$ algebra quotients of $R$. What algebras $A^{\prime}$ are parametrized by points $z_{A^{\prime}}$ in $(\overline{Z(E)}-Z(E))$ ? We answer this question when $r=2$, and $A^{\prime}$ has the same Hilbert function $H=H(E)$. Recall that $\Delta$ is the difference sequence of $H$, so $\Delta_{i}=h_{i-1}-h_{i}$. The sequence $\Delta(1)$ is $\Delta$ shifted one, $\Delta(1)_{i}=\Delta_{i+1}$. We say $\Delta(1) \geqslant E^{\prime}$ if each $\Delta_{i+1} \geqslant e_{i}^{\prime}$; and $E^{\prime}>E$ if each $e_{i}^{\prime} \geqslant e_{i}$ but some $e_{i}^{\prime}>e_{i}$.

Proposition 4.5 (Closures of $Z(E)$ ANd $G(E)$ When $r=2[21,24]$ ). When $r=2$, the varieties $Z(E)$ and $G(E)$ are open dense subsets of the irreducible varieties $Z_{H}$ and $G_{H}$ parametrizing all quotients of $R$ (or all graded quotients of $R$, respectively) having Hilbert function $H=H(E)$. Any noncompressed algebra of Hilbert function $H=H(E)$ has a socle type $E^{\prime}$ satisfying $\Delta(1) \geqslant E^{\prime}>E$. Any such sequence $E^{\prime}$ occurs as the socle type of an algebra $A^{\prime}$ in the closure.

When $r>2$, the question of the closure of $Z(E)$ in $Z_{H(E)}$ is quite open. Note that although $G_{H}$ is compact, $Z_{H}$ is not.

EXAMPLE 4.12. Limit $A^{\prime}$ of a family of compressed algebras, where the Hilbert function changes. Consider the flat family $\operatorname{Spec} A_{T} \rightarrow \operatorname{Spec} k[T]$, where $A_{T}=R[T] / I_{T}$ is the algebra

$$
k\left[\left[x_{1}, x_{2}\right]\right][T] /\left(T x_{1}^{3}+x_{1}^{2} x_{2}^{2}, x_{2}^{3}, x_{1}^{3} x_{2}, x_{1}^{4}\right) .
$$

Then when $t \neq 0$, the fibre $A_{t}$ is a compressed c.i. algebra with dual module generated by $f_{t}=4 y_{1}^{3}-3 t y_{1}^{2} y_{2}^{2}$, socle type $E_{4}=(0,0,0,0,1)$, and Hilbert function $H\left(E_{4}\right)=(1,2,3,2,1)$. When $t=0$, the fibre is $A_{0}=k\left[\left[x_{1}, x_{2}\right]\right] /\left(x_{2}^{3}, m^{4}\right)$ of socle type $(0,0,0,3)$, socle generators $y_{1}^{3}, y_{1}^{2} y_{2}, y_{1} y_{2}^{2}$ and Hilbert function $H^{\prime}=$ $(1,2,3,3,0)$.

The compressed algebras are the simplest algebras of a given socle type $E$ to construct. What Hilbert functions occur for noncompressed algebras of socle-type $E$ ? When $r=2$ a complete answer is known, extending the case $E^{\prime}$ above. We will consider first the simplest socle types, $E_{j}=\left(0, \ldots, 0, e_{j}, 0, \ldots\right)$ then sum the results. 
The sequences $H$ below all satisfy $H=\left(1,2, \ldots, v, h_{v}, \ldots, h_{j}, \ldots\right)$ with $v \geqslant h_{v} \geqslant$ $h_{v+1} \geqslant \cdots \geqslant 0$. Here $\Delta_{i}=\left(h_{i-1}-h_{i}\right)$; and $r=2$ (see $\left.\S 4 \mathrm{C}\right)$.

THEOREM 4.6A (GRADED ALGEBRAS OF SOCLE TYPE $E_{j}$ ). When $r=2$ the sequence $H$ is possible for the Hilbert function of a graded algebra of socle type $E_{j}$ iff $\Delta_{v} \leqslant \Delta_{v+1} \leqslant \cdots \leqslant \Delta_{j+1}=e_{j}$ and, of course, $h_{j+1}=h_{j+2}=\cdots=0$. The dimension of the irreducible variety $G(H ; E)$ parametrizing such graded algebras is

$$
\operatorname{dim}(G(H ; E))=e_{j}\left(j+1-e_{j}\right)=\sum_{i \leqslant j}\left(\Delta_{i+1}-\Delta_{i}\right)\left(i-h_{i-1}\right) .
$$

This dimension is just that of the Grassmannian $\operatorname{Grass}\left(e_{j}, R_{j}\right)$ diminished by the smallest number necessary for the matrices of partial derivatives of the dual generators to have nonmaximal rank $h_{i}$ in each degree. Thus the closure $\overline{G(H ; E)}$ can be considered a determinantal variety; it parametrizes all graded algebras $A^{\prime}$ of Hilbert function $H$. The socle type $E^{\prime}$ of any such algebra $A^{\prime}$ satisfies $\Delta(1) \geqslant E^{\prime} \geqslant E_{j}$, where $\Delta$ is the first difference of $H$.

THEOREM 4.6B (AlgeBRAS OF SOCLE TYPE $E_{j}$ ). When $r=2$, the sequence $H$ is possible for the Hilbert function of an algebra of socle type $E_{j}$ if $\Delta_{i} \leqslant e_{j}$ for all $i$, and $h_{j+1}=\cdots=0$. The dimension of the family $Z(H ; E)$ of all such algebras is $\operatorname{dim} Z\left(H ; E_{j}\right)=n-\Sigma\left(\Delta_{i}\right)\left(\Delta_{i}+1\right) / 2$. The closure $\left(\overline{Z(H ; E)} \cap Z_{H}\right)$ in $Z_{H}$ parametrizes all algebras of Hilbert function $H$ and having a socle type $E^{\prime}$ satisfying $\Delta(1) \geqslant E^{\prime} \geqslant E_{j}$.

THEOREM 4.6C (Algebras OF SOCLE TYPE $E$ WHEN $r=2$ ). The sequence $H$ is possible for the Hilbert function of a graded algebra of socle type $E$ iff for each $s$, $e_{s} \leqslant \Delta_{s+1} \leqslant \Delta_{s+2}+e_{s}$, and $H$ is eventually 0 . The sequence $H$ is possible for the Hilbert function of a nongraded algebra of socle type $E$ iff for each $s, e_{s} \leqslant \Delta_{s+1} \leqslant$ $\sum_{i \geqslant s} e_{i}$. The results concerning dimensions and closures in $Z_{H}$ are the same as those above, with $E$ replacing $E_{j}$.

Proof. The proofs are in [24]. The derivation from [24] of the characterization of $H$ in the general case is Proposition 4.2B in $\$ 4 \mathrm{C}$, plus the remark that, of course, $e_{s} \leqslant \Delta_{s+1}$. See also Proposition 4.2A, Theorem 4.10 of [21], and Theorem A.1B of the Appendix to [23] which answer-entirely analogously-a similar question for algebras defined by ideals $I$ of given generator orders.

When $r=3$, the Buchsbaum-Eisenbud structure theorem for height 3 Gorenstein algebras determines the possible Hilbert functions $H$, and the dimension of $G(H ; j)$ in the graded case of type 1 , socle degree $j$. The result concerning the possible Hilbert functions $H$ is implicit in [5] and is also specified in [32]; the dimension of the variety $G(H ; j)$ is implicit in [5]. When $r \geqslant 3$, little is known about $G(H ; E)$ and $Z(H ; E)$ in general.

The methods of this paper do allow us to construct certain other families of noncompressed algebras.

EXAMPLE 4.13. A family of noncompressed Gorenstein algebras. Suppose $r=3$ and consider the polynomial $f=y_{1}^{7}+F\left(y_{1}, y_{2}, y_{3}\right)$ where $F$ is a form of degree 4. It is easy to see, using Proposition 3.3 on $F$, that the Gorenstein algebra $A=R / \operatorname{Ann} f$ 
has in general the Hilbert function $H(A)=(1,3,6,3,1,1,1,1)$. A similar example when $F$ has degree 5 yields algebras of Hilbert function $H=(1,3,6,6,3,1,1,1)$, or in 4 variables with $F$ of degree $4, H=(1,4,10,4,1,1,1,1)$.

In particular this method of constructing the dual module could be used to study the Artin case of the stretched Gorenstein algebras of [30] (take $F$ of degree 2), as well as larger classes where $H$ terminates in a string of 1 's, or where $H$ and $H^{\prime}$ terminate similarly, $H>H^{\prime}$, and $Z_{H^{\prime}}$ is well understood.

Another class of noncompressed algebras can be constructed using sums of power of linear forms, as in Proposition 3.3. There we constructed the class of Gorenstein compressed algebras, whose dual modules can be added to the dual module of a given algebra, yielding new algebras of predictable Hilbert function. It is this additive property that is the key to constructing compressed algebras of arbitrary socle type. We here generalize that construction, beginning with a dual module $J F$ where $F$ is a sum of powers of $m$ linear forms; the resulting power sum Gorenstein algebras have the maximum possible Hilbert function bounded by $m$.

Proposition 4.7 (Power Sum Gorenstein algebras). Denote by $A_{B}$ the graded Gorenstein algebra $A_{B}=R / \operatorname{Ann} F$ where $F=\sum_{1}^{m} L_{i}^{j}$ and $L_{i}$ is the linear form $L_{i}=\sum_{k} b_{i k} y_{k}$. For coefficients $B=\left\{b_{i k}\right\}$ parametrized by a Zariski open subset of the affine space $\mathbf{A}^{r m}$, the algebra $A_{B}$ has Hilbert function $H\left(A_{B}\right)=H(m ; j)=$ $\left(1, h_{1}, \ldots, h_{j}=1,0, \ldots\right)$ where $h_{s}=\min \left(m, \# R_{s}, \# R_{j-s}\right)$.

Proof. It suffices to show that when $m \leqslant \min \left(\# R_{s}\right.$, \#R $\left.R_{j-s}\right)$ the matrix $B$ can be so chosen that $J^{u} F=L^{(s)} \stackrel{\text { def }}{=}\left\langle L_{1}^{s}, \ldots, L_{m}^{s}\right\rangle$, and that the length $\# L^{(s)}=m$. Corollary 3.2 shows that $\# L^{(s)}=m$ in general. The proof of Proposition 3.3 shows that the larger $n \times n$ matrix $M$ is in general nonsingular, so has a nonvanishing $m \times m$ minor determining a form $F=L_{1}^{j}+\cdots+L_{m}^{j}$ with $J^{j-s} F=L^{(s)}$. This completes the proof.

EXAMPle 4.14. Power sum Gorenstein algebra. If $r=4, j=7, m=5$ above, we obtain Gorenstein algebras of Hilbert function $H(5 ; 7)=(1,4,5,5,5,5,4,1)$. With $r=4, j=8, m=12$, the Hilbert function $H(12 ; 8)=(1,4,10,12,12,12,10,4,1)$. Note that the value $r=4$ is understood-but not explicitly noted-in $H(12 ; 8)$ above. If $r=3, H(12 ; 8)$ is $(1,3,6,10,12,10,6,3,1)$. (When $r=3$ the Hilbert functions and structure of graded Gorenstein algebras has been described in [9].)

We next show the additive property. The following definition eliminates superfluous summands.

Definition 4.3. Permissible sequence $(M ; J)$. The sequence of pairs $(M ; J)=\left(m_{1}\right.$; $\left.j_{1}\right), \ldots,\left(m_{n} ; j_{n}\right)$ is permissible for the Hilbert function bounds and socle degrees for Gorenstein algebras whose dual modules are added to that of the given algebra $A=R / I$, if $h_{s}(A)+\sum_{i} h_{s}\left(m_{i} ; j_{i}\right) \leqslant \# R_{s}$ for $s=j_{1}, \ldots, j_{n}$.

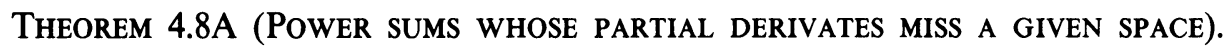
Given the vector spaces $V_{i} \subset \mathscr{R}_{i}$ satisfying $J^{1} V_{i} \subset V_{i-1}$ for $i=1, \ldots, j$, there is $a$ Zariski open set $U \subset \mathbf{A}^{r m}$ parametrizing ordered sets $L_{1}, \ldots, L_{m}$ of linear forms such that when $F=L_{1}^{j}+\cdots+L_{m}^{j}$ the module JF satisfies both (i) and (ii): 
(i) The degree-type $H(J F)=H(m ; j)($ see $§ 2 \mathrm{~A}(\mathrm{~d}))$.

(ii) For each $s$, the length $\#\left(J^{j-s} F \cap V_{s}\right)=\max \left(0, h_{s}(m, j)+\# V_{s}-\# R_{s}\right)$.

THEOREM 4.8B (ADDING DUAL MODULES OF POWER SUM GoRENSTEIN ALGEBRAS TO THAT OF A GIVEN ALGEBRA $A$ ). Given the algebra $A=R / I$ having dual module $\hat{A}=V \subset \mathscr{R}$, given a permissible sequence of pairs $(M ; J)$ as in Definition 4.3 , and given general power sum algebras $A_{1}=R / I(1), \ldots, A_{n}=R / I(n)$ having dual modules $A_{i}=J F_{i}$ where $F_{i}=\sum_{t=1}^{t=m_{i}} L_{i t}^{j_{i}}$ then the "sum" algebra $A^{\prime}=R / I^{\prime}$, where

$$
I^{\prime}=\operatorname{Ann}\left(V+\sum J F_{i}\right)=(I \cap I(1) \cap \cdots \cap I(n)),
$$

satisfies

(i) The Hilbert function $H\left(A^{\prime}\right)=H(A)+\sum H\left(m_{i} ; j_{i}\right)$ (see Definition 2.4A).

Proof. It suffices to prove Theorem 4.8A; then Theorem 4.8B follows just as Propositions 3.4 and 3.5 follow from Proposition 3.3. Given $V_{s}$ there are by Corollary 3.2 forms $L_{1}, \ldots, L_{m}$ such that the space $L^{(s)}=L_{1}^{s}, \ldots, L_{m}^{s}$ satisfies $\#\left(L^{(s)} \cap V_{s}\right)=\max \left(0, h_{s}(m, j)+\# V_{s}-\# R_{s}\right)$, so $L^{(s)}$ and $V_{s}$ intersect properly. For each $s$ there is a nonempty open subset $X_{s}$ of $\mathbf{A}^{r m}$ parametrizing such forms $L_{1}, \ldots, L_{m}$. Also, by Proposition 4.7 there is for each $s$ a nonempty open set $Y_{s} \subset \mathbf{A}^{m r}$ parametrizing $L_{1}, \ldots, L_{n}$ such that $J^{j-s} F=L^{(s)}$. The nonempty open set $U=\bigcap_{s}\left(X_{s} \cap Y_{s}\right)$ parametrizes ordered sets of linear forms satisfying the condition of Theorem 4.8A.

EXAMPLE 4.15. Type 2 power sum algebra. When $I=R, r=4$ and $A_{1}, A_{2}$ are the Gorenstein power sum algebras of Example 4.13, the "sum" is a type 2 power sum algebra $A^{\prime}$ having Hilbert function $H\left(A^{\prime}\right)=(1,4,10,17,17,17,14,5,1)$.

Note. The family of power sum algebras is not dense in $G(m ; j)$. The scheme $G(m ; j)$ parametrizing graded Gorenstein algebras of Hilbert function $H(m ; j)$, can be strictly larger in dimension than the family of power sum algebras. For example, suppose that in the compressed algebra case we take $m=\# \mathscr{R}_{j / 2}$. When $r=3$, the integer $m \cong(j / 2+1) \cdot(j / 2+2) / 2$, whereas

$$
\operatorname{dim} G(j)=\left(\# \mathscr{R}_{j}-1\right) \cong(j+2)(j+1) / 2 .
$$

Thus, $\operatorname{dim} G(j) \cong 4 m$, which is greater than $3 m$, the dimension of the parameter space $\mathbf{A}^{r m}$ for the linear forms $L_{1}, \ldots, L_{m}$. Note that by taking a large enough $m$ (instead of $m=\# R_{j / 2}$ ), we could indeed obtain an open dense set of the compressed graded Gorenstein algebras in question.

The methods of §3B do not immediately extend to give dimensions of the families of power sum algebras; they do yield some lower and upper bounds. Note also that a Gorenstein algebra $A$ having Hilbert function $H(m ; j)$ need not have a Gorenstein associated graded algebra $A^{*}$, in the noncompressed case: Proposition 3.7 does not extend.

4E. Extension to characteristic $p$. It is easy to see that the existence results of the paper are valid for arbitrary fields $k$ of characteristic 0 , such as the reals. By restating them using the algebra $\mathscr{D}$ of divided powers in place of $\mathscr{R}$, the results 
extend to characteristic $p$. The existence results require only an infinite field $k$; for the algebraic variety structure I assume also that $k$ is algebraically closed.

The restatement needed in characteristic $p$ is to replace the ring $\mathscr{R}$ by the divided power ring $\mathscr{D}=k\left[Y_{1}, \ldots, Y_{r}\right]$ where $Y_{i}^{(s)} Y_{i}^{(t)}=\left(\begin{array}{c}s+t \\ s\end{array}\right) Y_{i}^{(s+t)}$. Here, $\mathscr{D}_{s}$ has basis $Y_{1}^{(s)}, Y_{1}^{(s-1)} Y_{2}, \ldots, Y_{1}^{(s-2)} Y_{2}^{(2)}, \ldots, Y_{r}^{(s)}$. The action of $R$ on $\mathscr{D}$ is similar to its action on the ring $\mathscr{R}$, but without coefficients: $x_{i}^{t} \circ Y_{i}^{(s)}=Y_{i}^{(s-t)}$ if $s \geqslant t$, and is 0 otherwise. A fuller discussion is in the background section 1.4 of [2].

This requires changes in the statement and proof of the key Proposition 3.3. All statements involving powers $L^{j}$ of linear forms $L=b_{1} y_{1}+\cdots+b_{r} y_{r}$, become statements involving symbolic powers $L^{[j]}=b_{1}^{j} Y_{1}^{(j)}+b_{1}^{j-1} b_{2} Y_{1}^{(j-1)} Y_{2}+\cdots+$ $b_{r}^{j} Y_{r}^{(j)}$ in the divided power algebra.

Proof That det $M \neq 0$ IN GENERAL, AND OF COROLlaries 3.2 AND 3.3. It suffices to show $B=b_{i j}$ can be chosen so det $M^{\prime} \neq 0$. Considering the entries of $B$ as variables, the usual expansion $\operatorname{det} M=\sum(\operatorname{sign} \sigma) m_{1, \sigma(1)} \cdots m_{n, \sigma(n)}$ has terms that are all-different monomials in the $b_{i j}$ : we find that det $M$ is a nonidentically zero polynomial in the entries of $B$. Since $k$ is infinite, $B$ may be chosen so $\operatorname{det} M \neq 0$. This shows also that $\mathscr{D}_{u}$ is spanned by $L_{1}^{[u]}, \ldots, L_{n}^{[u]}$. This gives a characteristic-free proof of Corollary 3.2, and also completes the proof of Proposition 3.3 in characteristic $p$.

Thus, the change to divided powers changes the powers of linear forms used in the article, but little in the methods. However, the definition of a generalized sum of $s$ perfect $j$ th powers preceding Lemma 2.3 needs a change:

Definition 4.4. The form $F$ of degree $j$ in $\mathscr{D}=k\left[Y_{1}, Y_{2}\right]$ is a generalized sum of $s$ powers of linear forms iff $F=\sum F_{i}$ where each

$$
F_{i}=\sum_{k=1} a_{i k} L_{i}^{[j+1-k]} Y_{i}^{(k-1)}
$$

where $s=\sum s_{i}$, no $L_{i}$ depends on $L_{i^{\prime}}, i^{\prime} \neq i$, and where $Y_{i}=Y_{1}$ or $Y_{2}$, different from $L_{i}$. This completes the changes needed in the statements, for characteristic $p$. Of course, it is possible to use the divided powers also in characteristic 0 .

EXAMPLE 4.16. Compressed c.i. algebra (see Example 2.3). If $r=2$ and $F=Y_{1}^{[4]}+$ $Y_{2}^{[4]}+\left(Y_{1}+Y_{2}\right)^{[4]}$ the ideal $I=$ Ann $F \subset R$ is $I=\left(x_{1}^{2} x_{2}-x_{1} x_{2}^{2}\right)$ defining a compressed c.i. algebra $A=R / I$ of Hilbert function $H=(1,2,3,2,1)$.

$4 \mathrm{~F}$. Finding algebras of given type, number of generators $\mu(I)$, and embedding dimension three. J. Herzog and E. Kunz [35] have characterized which pairs of integers can occur as the deviation and type of a Cohen-Macaulay domain $A=R / I$; here $d=\mu(I)-(r-\operatorname{dim} A)$. D. Buchsbaum and D. Eisenbud showed in [9] that any odd number of generators $\mu(I) \geqslant 3$ could occur for the ideal defining a Gorenstein Artin algebra of embedding dimension 3. L. Avramov, using the latter result, has shown that all pairs $(e, \mu(I))$ greater than $(4,7)$, all $(e, 4)$ with $e \geqslant 2$, all $(3,2 n)$ above $(3,6)$, and all pairs $(2 n, 6)$ above $(4,6)$ occur for local Artin algebras of embedding dimension 3. I understand that $\mathrm{E}$. Ahmeeov has shown that all pairs $(e, 5)$ with $e \geqslant 2$ occur. Below it will be shown that all pairs $(2, \mu)$ with $\mu \geqslant 4$ occur. One can show similarly that all pairs $(3,2 n+1)$ when $n \geqslant 2$ occur; a method of L. 
Avramov then shows that the pairs $(2 n+1,6)$ occur. Thus all pairs $(e, \mu(I))$ above $(2,4)$ occur for Artin algebras of embedding dimension 3.

We will now see how compressed algebras and the more general power sum algebras can be used as a source of examples of Artin algebras having specified $(e, \mu(I), r)$, or having certain specified resolution skeletons. Given some specified data about the resolution of $A$ as an $R$-module, it is often easy enough to find socle types $E$, such that the simplest potential resolution of an Artin algebra $A$ having Hilbert function $H(E)$ fits the specified data. One then must check by calculating that a general enough compressed algebra of socle type $E$ has the specified data. The point is, this method can suggest algebras to fit numerical data. We use the method first to give examples of algebras of type $2, r=3$, with specified number of generators. Then we explore a question involving linking.

The results of this paper guarantee that, given $r$ and $E$ there are compressed algebras of the specified $r$ and $E$ having the Hilbert function $H(E)$. One must verify with an example that $\mu(I)$ can have the expected value. The existence of one such example shows that the general compressed algebra of the given socle type has the expected minimal $\mu(I)$ : having minimal $\mu(I)$ is a Zariski open condition on the irreducible variety $Z(E)$. To illustrate this method, we now list algebras of type 2 , embedding dimension 3 , having defining ideals generated by $5,6,7,8$, and 9 elements. Most of the algebras are compressed; the case $\mu(I)=7$ uses a power sum algebra.

EXAMPLE 4.17. Algebras of type $2, r=3$, with $\mu(I)=5$ to 9 . We use the duality of most of the article, with $R$ acting as derivatives on $\mathscr{R}$. We list $\mu(I), H(A), E(A)$, the generators $S$ for $\hat{A}$ in $\mathscr{R}$, and generators for $I$. The map $\sigma$ is the cyclic substitution $\sigma\left(x_{1}\right)=x_{2}, \sigma\left(x_{2}\right)=x_{3}, \sigma\left(x_{3}\right)=x_{1}$.

$\mu(I)=5: H=(1,3,4,1), E=(0,0,1,1), S=\left(y_{1} y_{2} y_{3}, y_{1}^{2}+y_{2}^{2}+y_{3}^{2}\right)$, and $I=$ $\left(x_{1}^{2}-x_{2}^{2}, x_{2}^{2}-x_{3}^{2}, x_{1}^{2} x_{2}, x_{1}^{2} x_{3}, x_{1} x_{3}^{2}\right)$.

$\mu(I)=6: \quad H=(1,3,6,4,1), \quad E=(0,0,0,1,1), \quad S=\left(y_{1}^{2} y_{2} y_{3}+y_{1} y_{2}^{2} y_{3}+\right.$ $\left.y_{1} y_{2} y_{3}^{2}, y_{1} y_{2} y_{3}\right)$, and $I=\left(x_{1}^{3}, x_{2}^{3}, x_{3}^{3}, f, \sigma f, \sigma^{2} f\right)$ where $f=x_{1}^{2} x_{2}-x_{1} x_{2}^{2}$.

$\mu(I)=7: H=(1,3,6,3,1), E=(0,0,1,0,1), A$ is a power sum algebra with pairs $M, J=(5,4)$ and $(2,2)$ so $H$ is the sum of Hilbert functions $(1,3,5,3,1)$ and $(1,2,1)$.

$$
S=\left(\left(y_{1}^{4}+y_{2}^{4}+y_{3}^{4}+\left(y_{1}+y_{2}\right)^{4}+\left(y_{1}+y_{3}\right)^{4}\right), y_{2} y_{3}\right),
$$

and

$$
\begin{gathered}
I=\left(x_{1} x_{2} x_{3}, x_{2} x_{3}^{2}, x_{2}^{2} x_{3}, x_{1}^{3}+x_{3}^{3}-x_{1} x_{2}^{2}-3 x_{1} x_{3}^{2},\right. \\
\left.x_{2}^{3}-x_{3}^{3}-2 x_{1} x_{2}^{2}+2 x_{1} x_{3}^{2}, x_{1}^{2} x_{2}-x_{1} x_{2}^{2}, x_{1}^{2} x_{3}-x_{1} x_{3}^{2}\right) . \\
\mu(I)=8: H=(1,3,6,2), E=(0,0,0,2), S=\left(y_{1} y_{2} y_{3}, y_{1}^{2} y_{2}+y_{2}^{2} y_{3}+y_{3}^{2} y_{1}\right), \text { and } \\
I=\left(x_{1}^{3}, x_{2}^{3}, x_{3}^{3}, x_{1} x_{2}^{2}, x_{1}^{2} x_{3}, x_{2} x_{3}^{2}, x_{1}^{2} x_{2}-x_{2}^{2} x_{3}, x_{2}^{2} x_{3}-x_{3}^{2} x_{1}\right) . \\
\begin{array}{c}
\mu(I)=9: H=(1,3,6,9,4,1), E=(0,0,0,0,1,1), S=\left(s\left(y_{1} y_{2}^{2} y_{3}^{2}\right), s\left(y_{1}^{4}\right)\right) \text { where } \\
s=1+\sigma+\sigma^{2}, \text { and }
\end{array} \\
\begin{array}{c}
I=\left(s\left(x_{2}^{2} x_{3}-x_{2} x_{3}^{2}\right), f, \sigma f, \sigma^{2} f, g, \sigma g, h, \sigma h, x_{1}^{2} x_{2} x_{3}-x_{1}^{2} x_{2}^{2}-x_{1}^{2} x_{3}^{2}\right) \\
\text { where } f=x_{1}^{3} x_{2}, g=x_{1}^{4}-x_{2}^{4}, h=x_{1} x_{2}^{3} .
\end{array}
\end{gathered}
$$


In the example below, we outline a proof that when $r=3$, a general compressed algebra of type 2 and socle degrees $(2 t, t+1)$ with $t \geqslant 1$ has $\mu(I)=2 t+2$, as in the case $\mu(I)=6$ above.

EXAMPLE 4.18. Compressed algebras with $(e, \mu(I))=(2,2 t+2)$ when $r=3$. Begin with a graded Gorenstein algebra $B=k[[x, y, z]] / I$ of socle degree $2 t$ such that $I$ has 2 generators $p_{1}, p_{2}$ of degree $t$ and $2 t-3$ generators of degree $t+1$. Such an algebra $B$ has Hilbert function

$$
H_{t}=\left(1,3,6, \ldots, \# R_{t-1}, \# R_{t}-2, \# R_{t-1}, \ldots, 6,3,1\right),
$$

and when $t>1$ is constructed by D. Buchsbaum and D. Eisenbud in [9] as follows (one modifies the example of Proposition 6.2 there as suggested on p. 467). When $t>1$ take $n=2 t-1$ and consider the $n \times n$ alternating matrix $C_{n}$ whose abovediagonal entries are zero except for

$$
C_{n}(i, j)= \begin{cases}x^{a(i, j)} & \text { if } i \text { is odd and } j=i+1, \\ y^{a(i, j)} & \text { if } i \text { is even and } j=i+1, \\ z^{a(i, j)} & \text { if } j=n-i+1\end{cases}
$$

Here $a(i, j)=3$ if both $i, j \leqslant 2 ; a(i, j)=2$ if exactly one of $i, j \leqslant 2$; and $a(i, j)=1$ otherwise. Let $p_{i}$ denote the Pfaffian of the minor of $C_{n}(i, i)$. Then $p_{1}=y^{t}, p_{n}=x^{t+1}$ and if $t>2, p_{t}=z^{t+1}+\cdots$ while if $t=2, p_{2}=z^{2}$. Thus $P f_{n-1}\left(C_{n}\right)$ has height 3 , and by Theorem 2.1 of [9] it is a Gorenstein ideal $I$ minimally generated by $p_{1}, \ldots, p_{n}$; it defines a Gorenstein algebra $B$ of Hilbert function $H_{t}$. Notice that $I \cap R_{t+1}=\left\langle R_{1}\left\langle p_{1}, p_{2}\right\rangle ; p_{3}, \ldots, p_{n}\right\rangle$. We define the algebra $A=R / I^{\prime}$ where $I^{\prime}=\left(W, p_{3}, \ldots, p_{n}\right)$ is generated by a colength one subspace of $I \cap R_{t+1}$. We choose $W$, a length-5 subspace of $R_{1}\left\langle p_{1}, p_{2}\right\rangle=\left\langle x p_{1}, \ldots, z p_{2}\right\rangle$ in such a way that $R_{1} W$ equals $R_{2}\left\langle p_{1}, p_{2}\right\rangle=\left\langle x^{2} p_{1}, \ldots, z^{2} p_{2}\right\rangle$, and that also no linear combination $h=c p_{1}+d p_{2}$ with $c, d \in k$ satisfies $\langle x h, y h, z h\rangle \subset W$. Each condition is Zariski open on $W \subset \mathbf{P}^{5}=P\left(R_{1}\left\langle p_{1}, p_{2}\right\rangle\right)$ and is easily satisfied. Then the algebra $A$ has type 2 , socle degrees $(2 t, t+1)$, has $\mu(I)=2 t+2$ all of degree $t+1$, and is compressed of Hilbert function

$$
H(A)=\left(1,3,6, \ldots, \# R_{t-1}, \# R_{t}, \# R_{t-1}+1, \# R_{t-2}, \ldots, 6,3,1\right) .
$$

Here $I_{s}^{\prime}=I_{s}$ for $s \geqslant t+2$.

A similar construction begins with a Gorenstein algebra $B=R / I$ of socle degree $2 t$ where $I$ has one generator of degree $t$ and $2 t$ of degree $t+1$ where $t \geqslant 2$. Taking $I^{\prime}=\left(I_{t+1}\right)$ yields a type 2 algebra $A=R / I^{\prime}$ of socle degrees $(2 t, t), \mu(I)=2 t+3$ all of degree $t+1$, and Hilbert function $H(A)=\left(1, \ldots, \# R_{t}, \# R_{t-1}, \ldots, 1\right) . A$ is - trivially-relatively compressed with respect to $B$. The case $\mu(I)=7$ in Example 4.17 suggests that when $r=3$, general enough power sum algebras of type 2 and pairs $(M, J)=\left(\left(\# R_{t}-1,2 t\right),(2, t)\right)$ should also have $\mu(I)=2 t+3$.

Larger types allow even more flexibility in constructing algebras of specified $\mu(I)$. Thus we can construct as above type 3 algebras $A$ of Hilbert function $H(2 t)$ where $I$ has $(2 t+3)$ generators all of degree $t+1$. We list below several algebras of type 3 and one of type 5 , including the lowest $\mu(I)$ of these types not hitherto covered. 
EXAMPLE 4.19. Compressed and power sum algebras of types 3 and $5, r=3$. We list $(e, \mu(I))$ first.

$(3,5): H=(1,3,6,5,1), E=(0,0,2,1)$. Compressed algebra with the expected number of generators $\mu(I)$.

$$
S=\left(y_{1}^{2} y_{2} y_{3}+y_{1} y_{2} y_{3}^{2}+y_{1} y_{2}^{2} y_{3}, y_{1}^{2} y_{3}+y_{2} y_{3}^{2}, y_{1} y_{3}^{2}+y_{1} y_{2}^{2}\right),
$$

and

$$
\begin{array}{r}
I=\left(x_{1}^{3}, x_{2}^{3}, x_{3}^{3}, x_{1}^{2} x_{2}-x_{1}^{2} x_{3}+x_{1} x_{3}^{2}-x_{1} x_{2}^{2}+x_{2} x_{3}^{2}-x_{2}^{2} x_{3},\right. \\
\left.x_{1} x_{2} x_{3}-2 x_{1}^{2} x_{2}+x_{1} x_{2}^{2}-x_{1} x_{3}^{2}-x_{2}^{2} x_{3}\right) .
\end{array}
$$

$(3,7): H=(1,3,6,5,1), E=(0,0,2,1)$. Compressed algebra with more than the expected number of generators $\mu(I)$.

$$
S=\left(y_{1}^{2} y_{2} y_{3}+y_{1} y_{2} y_{3}^{2}, y_{1} y_{2}^{2}, y_{2}^{2} y_{3}\right)
$$

and

$$
I=\left(x_{1}^{3}, x_{2}^{3}, x_{3}^{3}, x_{1} x_{2} x_{3}-x_{1}^{2} x_{2}-x_{2} x_{3}^{2}, x_{1}^{2} x_{3}-x_{1} x_{3}^{2}, x_{1} x_{2}^{2} x_{3}, x_{2}^{2} x_{3}^{2}\right) .
$$

$(3,7): H=(1,3,6,3,1)$. Take $M, J=((4,4),(2,2),(2,2))$. Power sum algebra with the expected number of generators $\mu(I)$.

$$
\begin{gathered}
S=\left(y_{1}^{4}+y_{2}^{4}+y_{3}^{4}+\left(y_{1}+y_{2}+y_{3}\right)^{4}, y_{1} y_{2}, y_{2} y_{3}\right) . \\
I=\left(f=x_{1}^{3}+x_{2}^{3}+x_{3}^{3}-4 x_{1} x_{2} x_{3}, u_{i}-x_{1} x_{2} x_{3}\right)
\end{gathered}
$$

where $u_{i}, i=1, \ldots, 6$, runs through the monomials of degree 3 not terms in $f$. This is the case $t=2$ of power sum algebras with type 3 , and $(M, J)=\left(\# R_{t}-2,2 t\right)$, $(2, t),(2, t)$, for which one expects $\mu(I)=2 t+3$.

$(5,6): H=(1,3,6,10,9,3), E=(0,0,0,0,2,3)$. This example behaves like a power sum algebra with $M, J=((4,5),(2,5),(2,5),(5,4)$ and $(4,4))$, and has the expected number of generators.

$$
\begin{gathered}
S=\left(y_{1}^{2} y_{2}^{3}+y_{1}^{3} y_{2}^{2}, y_{2}^{2} y_{3}^{3}+y_{2}^{3} y_{3}^{2}, y_{1}^{2} y_{2} y_{3}^{2}, 2 y_{1}^{3} y_{3}-3 y_{2}^{2} y_{3}^{2}, 2 y_{1} y_{3}^{3}-3 y_{1}^{2} y_{2}^{2}\right) . \\
I=\left(x_{1}^{4}, x_{2}^{4}, x_{3}^{4}, x_{1} x_{2}^{2} x_{3}, x_{1}^{3} x_{3}-x_{2}^{3} x_{3}+x_{2}^{2} x_{3}^{2}-x_{2} x_{3}^{3}\right. \\
\left.x_{1} x_{3}^{3}-x_{1}^{3} x_{2}+x_{1}^{2} x_{2}^{2}-x_{1} x_{2}^{3}\right) .
\end{gathered}
$$

In looking for Artin algebras not in the linking class of Gorenstein algebras, we tried type 2 algebras with $\mu(I)=5$, where the relations among the generators of $I$ could not be Koszul relations. Compressed algebras $A$ of socle type $E=(0,0,0,0,2)$ where $r=3$ have Hilbert function $(1,3,6,6,2,0)$ and expected resolution skeleton $0 \rightarrow R_{-7}^{2} \rightarrow R_{-5}^{6} \rightarrow R_{-3}^{4}+R_{-4}^{1} \rightarrow R \rightarrow A$, attained when $S=y_{1}^{2} y_{2} y_{3}, y_{1} y_{2}^{2} y_{3}+$ $y_{1} y_{2} y_{3}^{2}+y_{2} y_{3}^{3}$ and $I=\left(x_{1}^{3}, x_{2}^{3}, x_{3}^{3}-x_{1} x_{3}^{2}, x_{1} x_{2}^{2}+x_{2}^{2} x_{3}-x_{2} x_{3}^{2}, x_{3}^{4}\right)$-using the duality without coefficients of $\S 4 \mathrm{E}$. There are no Koszul relations in $R_{-5}^{6}$ since they would need degree at least 6 . J. Weyman pointed out that any algebra $A$ of this socle type is linked via the degree 3 generators of $I$ to algebras of lower Hilbert functions having Koszul relations; hence $A$ is in the linking class of Gorenstein algebras.

The following example singles out some compressed algebras whose linking class might be different. 
EXAMPLE 4.20. Candidate for compressed algebras linked to algebras of the same socle type. Consider compressed-and thin-algebras with 3 socle generators of degree 5 , in three variables (see $\$ 4 C$ ). They are in general defined by ideals $I$ having 6 generators of degree 4 , and have Hilbert function $H=(1,3,6,10,9,3,0)$. One expects the resolution $0 \rightarrow R_{-8}^{3} \rightarrow R_{-6}^{8} \rightarrow R_{-4}^{6} \rightarrow R \rightarrow A \rightarrow 0$. Linking by a general enough complete intersection $\left(f_{1}, f_{2}, f_{3}\right)$ in $I$ having initial degrees $(4,4,4)$ should yield algebras of the same socle type and Hilbert function. Note that the length of $A$ is 32 ; the dimension of the family $Z(E)$ of all compressed algebras of this socle type is $3((21-3)+(15-9))=72$. The dimension of the family of alignable algebras - quotients of $R$ that can be deformed to $k[x] /\left(x^{32}\right)$ is $2(31)=62$; thus there are nonalignable algebras in the family $Z(E)$ (see [18 and 23]). What is the linking class of these algebras?

We have discussed finding Artin algebras of given embedding dimension and Betti numbers. L. Avramov poses the more general problem of characterizing the possible sequences of Betti numbers for finite length modules over a regular local ring of dimension $r$.

\section{REFERENCES}

1. R. Achilles and L. Avramov, Relations between properties of a ring and its associated graded ring (Seminar Eisenbud, Singh, Vogel, vol. 2), Teubnertexte, no. 48, Teubner, Leipzig, 1982, pp. 5-29.

2. K. Akin, D. Buchsbaum and J. Weyman, Schur functors and Schur complexes, Adv. Math. 44 (1982), 207-270.

3. Barattero and Zatini, Relations between the type of a point on an algebraic variety and the type of its tangent cone, J. Algebra 66 (1980), 386-399.

4. D. Bayer, The division algorithm and the Hilbert scheme, Thesis, Harvard University, 1982.

5. D. Berman, Simplicity of a vector space of forms: finiteness of the number of complete Hilbert functions, J. Algebra 45 (1977), 52-57.

6. _ Hilbert functions of vector spaces of forms, Ph.D. Dissertation, University of Texas, Austin, 1978.

7. N Number of generators of a colength $n$ ideal, J. Algebra 73 (1981), 156-166.

8. J. Briançon, Description de $\operatorname{Hilb}^{n} \mathrm{C}\{x, y\}$, Invent. Math. 41 (1977), 45-89.

9. D. Buchsbaum and D. Eisenbud, Algebra structures for finite free resolutions, and some structure theorems for ideals of codimension 3, Amer. J. Math. 99 (1977), 447-485.

10. __ Almost linear resolutions, Appendix to D. Eisenbud and S. Goto, Linear free resolutions and minimal multiplicity, J. Algebra (to appear).

11. R. Buchweitz, Deformations de diagrammes, deploiements et singularités très rigides, liaison algébrique, Thèse, Universite de Paris VII, 1981.

12. J. Emsalem, Géométrie des points épais, Bull. Soc. Math. France 106 (1978), 399-416.

13. G. Gotzmann, Eine bedingung fur die Flachheit und das Hilbert-Polynom eines graduierten Ringes, Math. Z. 158 (1978), 61-70.

14. M. Granger, Géométrie des schémas de Hilbert ponctuels, Thèse, Université de Nice, 1981.

15. E. L. Green, Complete intersections and Gorenstein ideals, J. Algebra 52 (1978), 264-273.

16. J. H. Grace and A. Young, The algebra of invariants, Cambridge Univ. Press, New York, 1903; reprint, Chelsea, New York.

17. J. Herzog and E. Kunz, Der kanonische Modul eines Cohen-Macaulay-rings, Lecture Notes in Math., vol. 238, Springer-Verlag, Berlin, Heidelberg and New York, 1971.

18. A. Iarrobino and J. Emsalem, Some zero dimensional generic singularities: finite algebras having small tangent space, Compositio Math. 36 (1978), 145-188. (The matrix $M$ of $\$ 3.2$ and hence the example of $\$ 3.2$ are incorrect - see the Note following Example 4.1 of this article for a discussion.)

19. A. Iarrobino, Reducibility of the family of 0-dimensional schemes on a variety, Invent. Math. $\mathbf{1 5}$ (1972), 72-77.

20. , Number of generic singularities, Rice Univ. Stud. 59 (1972), 49-52. 
21. __ Punctual Hilbert schemes, Mem. Amer. Math. Soc. No. 188 (1977).

22. ___ Deformations of zero-dimensional complete intersections (after M. Granger, T. Gaffney; with Appendix, Global Hilbert scheme Hilb ${ }^{Q} P_{r}$ after Gotzmann), Proceedings of the Week of Algebraic Geometry, Bucharest 1980 (L. Badescu and H. Kurke, ed.), Teubnertexte, no. 40, Teubner, Leipzig, 1981, pp. 92-105.

23. ___ Deforming complete intersection Artin algebras. Appendix: Hilbert function of $\mathbf{C}[[x, y]] / I$, in Singularities, Proc. Sympos. Pure Math., vol. 40, Amer. Math. Soc., Providence, R. I., 1983, pp. 593-608.

24. __ Ancestor ideals of vector spaces of forms, preprint, 1975.

25. H. Kleppe, Deformations of schemes defined by vanishing of Pfaffians, J. Algebra 53 (1978), 84-92.

26. F. S. Macaulay, The algebraic theory of modular systems, Cambridge Univ. Press, New York, 1916.

27. , On a method of dealing with the intersections of plane curves, Trans. Amer. Math. Soc. 5 (1904), 385-410.

28. G. Mazzola, Generic finite schemes and Hochschild cocycles, Comment. Math. Helv. 55 (1980), 267-293.

29. D. Mumford, Pathologies. IV, Amer. J. Math. 97 (1975), 847-849.

30. J. Sally, Stretched Gorenstein rings, London Math. Soc. (2) 20 (1979), 19-26.

33. _ Number of generators of ideals in local rings, Dekker, New York, 1978.

32. R. Stanley, Hilbert functions of graded algebras, Adv. in Math. 28 (1978), 57-83.

33. E. K. Wakeford, On canonical forms, Proc. London Math. Soc. (2) 18 (1918), 403-410.

34. P. Schenzel, Über die freien Auflosungen extremaler Cohen-Macaulay-ringe, J. Algebra 64 (1980), 93-101.

35. J. Herzog and E. Kunz, On the deviation and the type of a Cohen-Macaulay Ring, Manuscripta Math. 9 (1973), 383-388.

36. D. Anick, Thin algebras of embedding dimension three, preprint, M.I.T., 1984.

37. R. Fröberg and D. Laksov, Extremal Cohen-Macaulay rings, and Gorenstein rings, preprint, Stockholm University (to appear).

38. J. Elias and A. Iarrobino, Extremal Gorenstein algebras of codimension three. Appendix: Hilbert function of compressed algebras, preprint, 1984.

DePartment of Mathematics, Northeastern University, Boston, MassachuSETTS 02115 